\title{
Spectral curves in gauge/string dualities: integrability, singular sectors and regularization.
}

\author{
Boris Konopelchenko $^{1}$, Luis Martínez Alonso ${ }^{2}$ \\ and Elena Medina ${ }^{3}$ \\ ${ }^{1}$ Dipartimento di Matematica e Fisica "Ennio De Giorgi", Universitá del Salento \\ and Sezione INFN, 7310 Lecce, Italy \\ ${ }^{2}$ Departamento de Física Teórica II, Facultad de Ciencias Físicas, Universidad \\ Complutense, 28040 Madrid, Spain \\ ${ }^{3}$ Departamento de Matemáticas, Facultad de Ciencias, Universidad de Cádiz, 11510 \\ Puerto Real, Cádiz, Spain
}

\begin{abstract}
We study the moduli space of the spectral curves $y^{2}=W^{\prime}(z)^{2}+f(z)$ which characterize the vacua of $\mathcal{N}=1 U(n)$ supersymmetric gauge theories with an adjoint Higgs field and a polynomial tree level potential $W(z)$. The integrable structure of the Whitham equations is used to determine the spectral curves from their moduli. An alternative characterization of the spectral curves in terms of critical points of a family of polynomial solutions $\mathbb{W}$ to Euler-Poisson-Darboux equations is provided. The equations for these critical points are a generalization of the planar limit equations for one-cut random matrix models. Moreover, singular spectral curves with higher order branch points turn out to be described by degenerate critical points of $\mathbb{W}$. As a consequence we propose a multiple scaling limit method of regularization and show that, in the simplest cases, it leads to the Painlevè-I equation and its multi-component generalizations.
\end{abstract}

PACS numbers: 02.30.lk, 11.15.-q, 02.10.Yn

J. Phys. A: Math. Theor. 46 (2013) 
Spectral curves in gauge/string dualities: integrability, singular sectors and regularization. 2

\section{Introduction}

In a series of seminal papers [1-3] Cachazo, Dijkgraaf, Intriligator and Vafa proved that a class of hyperelliptic curves of the form

$$
y^{2}=W^{\prime}(z)^{2}+f(z),
$$

where $W(z)$ and $f(z)$ are polynomials such that $\operatorname{deg} f=\operatorname{deg} W-2$, are essential to study the low energy dynamics of pure $\mathcal{N}=2 U(n)$ supersymmetric gauge theories deformed to $\mathcal{N}=1$ by a tree level potential $\operatorname{Tr} W(\Phi)$ for the Higgs field $\Phi$. These curves emerge from the duality between supersymmetric gauge theories and string theories on deformed 3-dimensional Calabi-Yau (CY) manifolds defined by the equation

$$
W^{\prime}(z)^{2}+f(z)+u^{2}+v^{2}+w^{2}=0 .
$$

Here the polynomial $f(z)$ is introduced to regularize the singular CY manifold

$$
W^{2}+u^{2}+v^{2}+w^{2}=0
$$

according to the process called Geometric Transition [1]. The curves (1) can be also generated from a special class of Seiberg-Witten curves [4] of pure $\mathcal{N}=2$ gauge theories which satisfy a certain factorization property [5].

Curves of the form (1) are relevant in the study of the asymptotic eigenvalue distribution as $n \rightarrow \infty$ of matrix models with partition functions

$$
Z_{n}=\int e^{-n \operatorname{Tr} W(M)} \mathrm{d} M,
$$

where $n$ is the matrix dimension. The best known matrix model is the Hermitian model [6.7], but more general models based on sets of matrices such that the eigenvalues are constrained to lie on some fixed path $\Gamma$ in the complex plane are used in the analysis of gauge/string dualities [8-11]. The curves (1) also arise in the study of the asymptotic zero distribution as $n \rightarrow \infty$ of monic orthogonal polynomials $P_{n}(z)=z^{n}+\cdots$, verifying the conditions 12 19

$$
\int_{\Gamma} P_{n}(z) z^{k} e^{-n W(z)} \mathrm{d} z=0, \quad k=0, \ldots, n-1,
$$

on a certain path $\Gamma$ in the complex plane.

The curves (1) have an even number $2 q(1 \leq q \leq N)$ of branch points given by the odd-order roots $\boldsymbol{\beta}=\left(\beta_{1}, \ldots, \beta_{2 q}\right)$, of the polynomial $y(z)^{2}$. In the applications to gauge/string dualities the curves (1) are characterized by imposing $q$ period relations of the form

$$
\oint_{A_{j}} y(z) \mathrm{d} z=-4 \pi \text { i } s_{j}, \quad j=1, \ldots, q,
$$

where $\mathbf{s}=\left(s_{1}, \ldots, s_{q}\right)$ are $q$ given arbitrary complex numbers called 't Hooft parameters. Here $y(z)$ is the branch of (1) with asymptotic behavior

$$
y(z)=W^{\prime}(z)+\mathcal{O}\left(z^{-1}\right), \quad z \rightarrow \infty,
$$


Spectral curves in gauge/string dualities: integrability, singular sectors and regularization. 3

and $A_{j}$ are counterclockwise cycles around $q$ disjoint oriented curves (cuts) $\gamma_{j}$ with endpoints $\left(\beta_{2 j-1}, \beta_{2 j}\right)$.

The present work is a natural continuation of our study of phase transitions in random matrix models $[8]$ and in the space of vacua in supersymmetric gauge theories [20]. Our main now is to study the structure of the moduli space of the curves (1) which satisfy a system of period relations of the form (5). We assume that

$$
W(z)=\frac{z^{N+1}}{N+1}+\sum_{n=1}^{N} g_{n} z^{n}, \quad f(z)=\sum_{k=1}^{N} z^{N-k} t_{k},
$$

where the coefficients $g_{k}$ are fixed while the coefficients $t_{k}$ are considered as arbitrary varying complex parameters. Henceforth we will refer to (1) as spectral curves and to the coefficients $\mathbf{t}=\left(t_{1}, \ldots, t_{N}\right)$ as the deformation parameters. Our main interest is to apply the theory of integrable systems to describe the analyticity properties of the roots of $y(z)^{2}$ as functions of either the 't Hooft parameters or the deformation parameters.

From the point of view of the underlying $U(n)$ gauge theory, $q$-cut spectral curves are associated to classical vacua such that the $n$ eigenvalues of the Higgs field $\Phi$ distribute among $q$ critical points $a_{j}$ of $W(z)$ with multiplicities $n_{j}$ where $n_{1}+\ldots+n_{q}=n$. On these vacua the gauge group is spontaneously broken into $q$ factors

$$
U(n) \rightarrow U\left(n_{1}\right) \times U\left(n_{2}\right) \times \cdots \times U\left(n_{q}\right) .
$$

The role of spectral curves in the low energy dynamics of the $U(n)$ gauge theory is of central importance since they determine the prepotential functional (see [1], [2] and [3])

$$
\mathcal{F}=\int_{\gamma} \rho(z) W(z)|\mathrm{d} z|-\frac{1}{2} \int_{\gamma} \int_{\gamma} \rho(z) \rho\left(z^{\prime}\right) \log \left(z-z^{\prime}\right)^{2}|\mathrm{~d} z|\left|\mathrm{d} z^{\prime}\right|,
$$

which is the key object to obtain the vacuum expectation values of many important operators of the quantum theory. Here $\gamma$ denotes the union of all the cuts

$$
\gamma=\gamma_{1} \cup \cdots \cup \gamma_{q},
$$

and the function $\rho(z)$ (the spectral density) is defined by

$$
\rho(z)|\mathrm{d} z|=\frac{y\left(z_{+}\right) \mathrm{d} z}{2 \pi \mathrm{i}}=-\frac{y\left(z_{-}\right) \mathrm{d} z}{2 \pi \mathrm{i}}, \quad z \in \gamma .
$$

The subscripts \pm in $z_{ \pm}$indicate the limits of functions from the left and right of the oriented cuts. The 't Hooft parameters can be considered as partial charges with respect to the spectral density since we may rewrite (5) as

$$
\int_{\gamma_{j}} \rho(z)|\mathrm{d} z|=s_{j} .
$$

The spectral density obviously depends on the choice of the cuts $\gamma_{j}$. However, as it is known in the study of gauge/string dualities [1]- [3], the prepotential as a function of the 't Hooft parameters is determined by the periods of the differential $y(z) \mathrm{d} z$ on a certain set of non-compact cycles in (1) (special geometry relations). As a consequence $\mathcal{F}(\mathbf{s})$ is invariant under deformation of the cuts in the same homology classes of $\mathbb{C} \backslash\left\{\beta_{i}\right\}_{i=1}^{2 q}$. 
Spectral curves in gauge/string dualities: integrability, singular sectors and regularization. 4

In the present work we introduce a decomposition of the moduli space of spectral curves onto classes in terms of sets $\mathcal{M}_{q}$ of spectral curves with at most $2 q$ branch points. Each class $\mathcal{M}_{q}$ is naturally stratified into a regular and a singular sector. The regular sectors contain the spectral curves which generically are determined by the constraints (5), so that they depend on $q$ moduli which may be either the 't Hooft parameters or a subset of $q$ deformation parameters. In the language of supersymmetric gauge theory [21], the regular sectors represent the different quantum phases of the vacua of the gauge model and, consequently, the singular sectors describe the regions of phase transitions [20].

We revisit the theory of Whitham equations for spectral curves 22 to find the differential equations which characterize the classes $\mathcal{M}_{q}$ and to determine the spectral curves from systems of implicit equations involving the branch points of $y(z)^{2}$ and both the 't Hooft and the deformation parameters. We also give a simple proof of the $\tau$-function character of $\log \mathcal{F}$. Furthermore, we provide an alternative way to characterize the spectral curves (1) from of a reduced system of implicit equations for the branch points involving the $q$ deformation parameters $\left(t_{1}, \ldots, t_{q}\right)$ only. The reduced system represents the critical points of certain polynomial solutions $\mathbb{W}$ of Euler-PoissonDarboux equations [25], and it turns out to be a generalization for multicut spectral curves of the planar limit equations of one-cut $(q=1)$ random matrix models [26]27]. The main advantage of the use of reduced systems is that they drastically simplify the analysis of singular sectors where the gradient catastrophe for the branch points and, consequently, the breaking of analyticity for the spectral curve and the free energy (8) happens. In this way we show how singular spectral curves corresponding to the emergence of higher order branch points can be described in terms of simple classes of degenerate critical points of the solutions $\mathbb{W}$ of the Euler-Poisson-Darboux equations. Moreover, the reduced systems leads naturally to the multiple scaling limit method to regularize the critical behavior of the branch points. Thus we propose a method of regularization of the gradient catastrophes based on substituting the critical point equations for the functions $\mathbb{W}$ by the Euler-Lagrange equations for a density functional $\mathbb{W}^{\text {reg }}$ obtained by adding appropriate terms with derivatives to $\mathbb{W}$. In the simplest cases the corresponding regularizing differential equations are the Painlevè-I equation and its multi-component extensions.

The paper is organised as follows. In section 2 we analyze the moduli space of the spectral curves and define the classes $\mathcal{M}_{q}$ as well as their regular and singular sectors. We present a method to determine spectral curves from the set of 't Hooft parameters and introduce the notions of spectral density and the prepotential functional. Then we analyze the integrable structure supplied by Whitham equations with respect to 't Hooft parameters. Section 3 deals with the analysis of the moduli space of spectral curves in terms of deformation parameters and with the study of the analyticity properties of the roots of $y(z)^{2}$ as functions of the deformation parameters. We illustrate our theoretical scheme with a complete description of the moduli space of spectral curves 
Spectral curves in gauge/string dualities: integrability, singular sectors and regularization. 5

for the Gaussian and cubic models

$$
W(z)=\frac{z^{2}}{2}, \frac{z^{3}}{3}-g z .
$$

Section 4 presents the method of reduced systems based on solutions of Euler-PoissonDarboux equations to determine spectral curves. We also show the connection between singular spectral curves and degenerate solutions of reduced systems. Sections 5 and 6 describe a simple implementation of the multiple scaling limit to regularize gradient catastrophes of the branch point functions $\boldsymbol{\beta}(\mathbf{t})$. Moreover, the natural emergence of the Painlevè-I equation is demonstrated.

\section{The moduli space of spectral curves}

We may distinguish different classes in the set of spectral curves (1) by looking at the multiplicities $\left[m_{i_{1}}, m_{i_{2}}, \ldots\right],\left(1 \leq i_{1}<i_{2}<\ldots\right)$ of the roots of $y(z)^{2}$, where $m_{i}$ is the number of roots with multiplicity equal to $i$. The polynomial $y(z)^{2}$ is of order $2 N$, so that it has an even number $2 q$ of roots $\beta_{i}$ with odd multiplicities (branch points of $y(z)$ ) and a certain number $p$ of roots $\alpha_{l}$ with even multiplicities.

As we said in the introduction, the spectral curves (1) arising in the study of gauge/string dualities are determined imposing $q$ period conditions (5). Thus we will henceforth concentrate on families of spectral curves such that $y(z)^{2}$ is of the form

$$
y^{2}(z)=\prod_{l=1}^{p}\left(z-\alpha_{l}\right)^{2} \prod_{i=1}^{2 q}\left(z-\beta_{i}\right), \quad p+q=N .
$$

Indeed these families depend generically on $q$ free parameters since equating (1) and (11), then identifying coefficients of powers of $z$ leads to a system of $2 N$ equations for determining the $2 N+q$ variables $\left(\alpha_{l}, \beta_{i}, t_{k}\right)$. We will see that the moduli space of the spectral curves (1) which satisfy (5) and are of the form (11) can be described in terms of the set of 't Hooft parameters $\mathbf{s}=\left(s_{1}, \ldots, s_{q}\right)$.

\subsection{Regular and singular sectors of spectral curves}

In order to deal with the structures of singularities we will consider degenerations of the factorization (11) in which some roots coalesce. This motivates the following definition:

Definition 1 Given $q=0,1, \ldots, N$ we denote by $\mathcal{M}_{q}$ the set of all the spectral curves which have $2 q$ branch points at most.

The class $\mathcal{M}_{q}$ represents the set of all spectral curves which admit a factorization of the form (11) where some of the roots $\left(\alpha_{l}, \beta_{i}\right)$ may coincide. Obviously we have

$$
\mathcal{M}_{0} \subset \mathcal{M}_{1} \cdots \subset \mathcal{M}_{N}
$$

Definition 2 We define the regular sector $\operatorname{reg} \mathcal{M}_{q}$ as the subset of the spectral curves in $\mathcal{M}_{q}$ which have all the roots $\left(\alpha_{l}, \beta_{i}\right)$ distinct. We define the singular sector of $\mathcal{M}_{q}$ by $\operatorname{sing} \mathcal{M}_{q}=\mathcal{M}_{q} \backslash \operatorname{reg} \mathcal{M}_{q}$. 
Spectral curves in gauge/string dualities: integrability, singular sectors and regularization. 6

The regular sector $\operatorname{reg} \mathcal{M}_{q}$ represents the set of spectral curves such that $y(z)^{2}$ factorizes in the form (11) with distinct roots $\left(\alpha_{l}, \beta_{i}\right)$. The spectral curves in the singular sector sing $\mathcal{M}_{q}$ admit a factorization of the form (11) but such that some of the roots $\left(\alpha_{l}, \beta_{i}\right)$ coincide. It is clear that $\mathcal{M}_{q^{\prime}} \subset \operatorname{sing} \mathcal{M}_{q}$ for $q^{\prime}<q$.

\subsection{Determination of spectral curves from 't Hooft parameters}

To determine spectral curves of the form (11) which satisfy the period relations (5) we notice that the condition (6) implies

$$
\prod_{l=1}^{p}\left(z-\alpha_{l}\right)=\left(\frac{W^{\prime}(z)}{y_{0}(z)}\right)_{\oplus},
$$

where $y_{0}(z)$ is the function

$$
y_{0}(z)=\sqrt{\prod_{i=1}^{2 q}\left(z-\beta_{i}\right)},
$$

such that $y_{0}(z) \sim z^{q}$ as $z \rightarrow \infty$ and $\oplus$ stands for the sum of the nonnegative powers of the Laurent series of $W^{\prime}(z) / y_{0}(z)$ at infinity. Then from (13) we may determine the double roots $\alpha_{l}$ as functions of the simple roots $\beta_{i}$. Hence the function $y^{2}(z)$ is completely determined by the set of simple roots $\beta_{i}$.

The simple roots $\beta_{i}$ can be determined as follows. We first substitute

$$
y(z)=\left(\frac{W^{\prime}(z)}{y_{0}(z)}\right)_{\oplus} y_{0}(z)
$$

in (1) and identify the coefficients of $1, z, \cdots, z^{N+q-1}$ in both members of (1). The remaining coefficients do not give independent relations because as $z \rightarrow \infty$.

$$
y(z)^{2}-W^{\prime}(z)^{2}=\left(\frac{W^{\prime}(z)}{y_{0}(z)}\right)_{\ominus}\left[\left(\frac{W^{\prime}(z)}{y_{0}(z)}\right)_{\ominus} y_{0}(z)-2 W^{\prime}(z)\right] y_{0}(z)=\mathcal{O}\left(z^{N+q-1}\right) .
$$

Thus we find $N+q$ equations for the $N+2 q$ variables $\left(\beta_{i}, t_{k}\right)$. The required $q$ additional independent relations are provided by the period conditions

$$
\oint_{A_{j}} y(z) \mathrm{d} z=-4 \pi \mathrm{i} s_{j}, \quad j=1, \ldots, q,
$$

where $\mathbf{s}=\left(s_{1}, \ldots, s_{q}\right)$ are the 't Hooft parameters and $A_{j}$ are counterclockwise cycles around fixed cuts $\gamma_{j}$ connecting the pairs $\left(\beta_{2 j-1}, \beta_{2 j}\right)$ of branch points. It is clear that the period conditions only depend on the homology classes of the cuts in $\mathbb{C} \backslash\left\{\beta_{i}\right\}_{i=1}^{2 q}$. Hence the natural choice is to take each cut $\gamma_{j}$ in the homology class of the straight line segment $\left[\beta_{2 j-1}, \beta_{2 j}\right]$, so that 15 can be rewritten as

$$
\int_{\beta_{2 j-1}}^{\beta_{2 j}} y\left(z_{+}\right) \mathrm{d} z=2 \pi \mathrm{i} s_{j}, \quad j=1, \ldots, q .
$$

In this way we have a method for determining the $N+2 q$ variables $\left(\beta_{i}, t_{k}\right)$ as functions of the 't Hooft parameters by means of a system of $N+2 q$ implicit equations. Moreover, 
Spectral curves in gauge/string dualities: integrability, singular sectors and regularization. 7

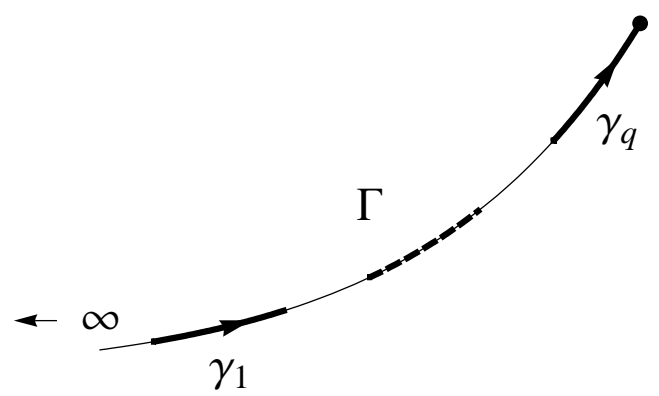

Figure 1. The semi-infinite path $\Gamma$ ending at $\beta_{2 q}$.

from (16) it is clear that for $q>1$ the set of solutions provided by this method depends on how we split the $2 q$ simple roots into pairs to define the cuts. In the applications to gauge/string dualities each of these solutions characterizes a different classical limit $\mathbf{s} \rightarrow \mathbf{0}$. In particular if the deformation parameters of a solution vanish as $\mathbf{s} \rightarrow \mathbf{0}$ then the polynomial $f$ vanishes and the spectral curve reduces to $y^{2}=W^{\prime}(z)^{2}$. Thus, the $2 q$ simple roots of the spectral curve corresponding to this solution represent a regularization of $q$ double roots of the undeformed curve.

In general, as we will see below in the analysis of the cubic model, this method for determining the cut endpoints leads to several families of solutions $\left(\beta_{i}(\mathbf{s}), t_{k}(\mathbf{s})\right)$ which determine several families of spectral curves in $\operatorname{reg} \mathcal{M}_{q}$.

\subsection{The spectral density}

Let us consider a spectral curve in the regular sector $\operatorname{reg} \mathcal{M}_{q}$ and take a semi-infinite oriented regular path $\Gamma$ (see figure 1) containing all the cuts $\gamma_{j}$. Henceforth we will assume that $\Gamma$ admits a parametrization $z(t)=(x(t), y(t))$ such that at least one of the components is a strictly monotone function of $t$. For each $z^{\prime}$ in $\Gamma$ let us denote by $\Gamma_{z^{\prime}}$ the semi-infinite $\operatorname{arc}$ of $\Gamma$ ending at $z^{\prime}$ and define

$$
\log \left(z-z^{\prime}\right)=\log \left|z-z^{\prime}\right|+\int_{\Gamma_{z, z^{\prime}}} \frac{\mathrm{d} u}{u-z^{\prime}}, \quad z^{\prime} \in \Gamma, \quad z \in \mathbb{C} \backslash \Gamma_{z^{\prime}},
$$

where $\Gamma_{z, z^{\prime}}$ is any path in $\mathbb{C} \backslash \Gamma_{z^{\prime}}$ connecting $z^{\prime}+\left|z-z^{\prime}\right|$ to $z$. It is clear that $\log \left(z-z^{\prime}\right)$ depends analytically of $z$ in $\mathbb{C} \backslash \Gamma_{z^{\prime}}$. For example if $\Gamma$ is a real interval of the form $\left(-\infty, x_{0}\right]$ then (17) determines the principal branch of $\log \left(z-z^{\prime}\right)$. Let us now introduce the function

$$
g(z)=\int_{\gamma} \log \left(z-z^{\prime}\right) \rho\left(z^{\prime}\right)\left|\mathrm{d} z^{\prime}\right|, \quad \gamma=\gamma_{1} \cup \cdots \cup \gamma_{q},
$$

where $\rho(z)$ is the spectral density

$$
\rho(z)|\mathrm{d} z|=\frac{y\left(z_{+}\right) \mathrm{d} z}{2 \pi \mathrm{i}}=-\frac{y\left(z_{-}\right) \mathrm{d} z}{2 \pi \mathrm{i}}, \quad z \in \gamma .
$$


Spectral curves in gauge/string dualities: integrability, singular sectors and regularization. 8

Note that both $y(z)-W^{\prime}(z)$ and $-2 g^{\prime}(z)$ are analytic in $\mathbb{C} \backslash \gamma$, vanish as $z \rightarrow \infty$ and, according to (9), have the same jump on $\gamma$. Therefore we obtain the identity

$$
y(z)=W^{\prime}(z)-2 g^{\prime}(z)=W^{\prime}(z)-2 \int_{\gamma} \frac{\rho\left(z^{\prime}\right)}{z-z^{\prime}}\left|\mathrm{d} z^{\prime}\right|
$$

which determines the spectral curve from the spectral density. It also implies the asymptotic behavior

$$
y(z)=W^{\prime}(z)-2 g^{\prime}(z)=W^{\prime}(z)-\frac{2 s}{z}+\mathcal{O}\left(z^{-2}\right), \quad \text { as } z \rightarrow \infty,
$$

where $s$ denotes the so called total t'Hooft parameter

$$
s=s_{1}+\cdots+s_{q} .
$$

Moreover, using

$$
y\left(z_{+}\right)+y\left(z_{-}\right)=0, \quad z \in \gamma,
$$

we get

$$
W^{\prime}(z)-\left(g^{\prime}\left(z_{+}\right)+g^{\prime}\left(z_{-}\right)\right)=0, \quad z \in \gamma .
$$

This equation means that $W(z)-\left(g\left(z_{+}\right)+g\left(z_{-}\right)\right)$is constant on each connected piece of $\gamma$ or, equivalently, that there are (not necessarily equal) complex numbers $l_{i}$ such that

$$
W(z)-\left(g\left(z_{+}\right)+g\left(z_{-}\right)\right)=l_{i}, \quad \forall z \in \gamma_{i} .
$$

The identities (24) are essential for the applications of spectral curves. There are two particularly important examples:

- The spectral curves arising in the large $n$-limit of Hermitian matrix models (3) lead to normalized spectral densities $\rho(z)$ which represent the asymptotic eigenvalue distribution of the models and are supported on a finite number of disjoint real intervals 6, 7]. In the electrostatic interpretation $\rho(x)$ describes the equilibrium density of a charge distribution on the real line $\mathbb{R}$ under the action of an external real potential $W(x)$. In this case all the parameters $l_{i}(24)$ are equal and represent the constant value of the total electrostatic potential on the support $\gamma$ of the equilibrium density $\rho(x)$.

- The families of orthogonal polynomials on curves $\Gamma$ satisfying the so-called $S$ property (see for example [12], [13], [18] and [19]) determine spectral curves with associated spectral densities $\rho(z)$ which represent the asymptotic distribution of the zeros of the polynomials and are supported on a finite number of finite disjoint curves contained in $\Gamma$. In the electrostatic interpretation $\rho(x)$ describes the equilibrium density of a charge distribution on $\Gamma$ under the action of the external real potential $\operatorname{Re} W$. In this case all the parameters $l_{i}(24)$ have the same real part since this common real part represents the constant value of the total electrostatic potential on the support $\gamma$ of the equilibrium density $\rho(x)$.

The class of spectral curves used in the study of gauge/string dualities generalizes those arising in Hermitian models and families of orthogonal polynomials. 
Spectral curves in gauge/string dualities: integrability, singular sectors and regularization. 9

\subsection{The prepotential}

The prepotential associated to a spectral curve is defined by

$$
\mathcal{F}=\int_{\gamma} \rho(z) W(z)|\mathrm{d} z|-\frac{1}{2} \int_{\gamma} \int_{\gamma} \rho(z) \rho\left(z^{\prime}\right) \log \left(z-z^{\prime}\right)^{2}|\mathrm{~d} z|\left|\mathrm{d} z^{\prime}\right|,
$$

where we assume that $\log \left(z-z^{\prime}\right)^{2}$ is given by

$$
\log \left(z-z^{\prime}\right)^{2}=\log \left(z_{+}-z^{\prime}\right)+\log \left(z_{-}-z^{\prime}\right), \quad z, z^{\prime} \in \Gamma .
$$

The consistence of (26) requires that

$$
\log \left(z_{+}-z^{\prime}\right)+\log \left(z_{-}-z^{\prime}\right)=\log \left(z_{+}^{\prime}-z\right)+\log \left(z_{-}^{\prime}-z\right), \forall z \neq z^{\prime} \text { in } \Gamma,
$$

where the subscripts \pm in $z_{ \pm}$indicate the limits of functions from the left and right of the oriented curve $\Gamma$. It is easy to prove that the property $(27)$ is satisfied if $\Gamma$ verifies our previous assumption that at least one of the components of the parametrization $z=(x(t), y(t))$ is a strictly monotone function of $t$. For example if $\Gamma$ is a real interval of the form $\left(-\infty, x_{0}\right]$ then (17) determines the principal branch of $\log \left(z-z^{\prime}\right)$ and $(26)$ yields $\log \left(z-z^{\prime}\right)^{2}=2 \log \left|z-z^{\prime}\right|$.

The property (27) is required to prove that the prepotential satisfies the important relations

$$
\frac{\partial \mathcal{F}}{\partial s_{i}}=l_{i} .
$$

Indeed, from (24)- (25), taking into account that $\rho\left(\beta_{j}\right)=0$, we have

$$
\begin{aligned}
\frac{\partial \mathcal{F}}{\partial s_{i}} & =\int_{\gamma}\left(W(z)-\int_{\gamma} \log \left(z-z^{\prime}\right)^{2} \rho\left(z^{\prime}\right)\left|\mathrm{d} z^{\prime}\right|\right) \frac{\partial \rho(z)}{\partial s_{i}}|\mathrm{~d} z| \\
& =\int_{\gamma}\left(W(z)-\left(g\left(z_{+}\right)+g\left(z_{-}\right)\right)\right) \frac{\partial \rho(z)}{\partial s_{i}}|\mathrm{~d} z| \\
& =\sum_{j=1}^{q} l_{j} \int_{\gamma_{j}} \frac{\partial \rho(z)}{\partial s_{i}}|\mathrm{~d} z|=\sum_{j=1}^{q} l_{j} \frac{\partial s_{j}}{\partial s_{i}}=l_{i} .
\end{aligned}
$$

From the definition (19) of the spectral density it is clear that $\rho(z)$ depends on the choice of the cuts $\gamma_{j}$ connecting the pairs $\left(\beta_{2 j-1}, \beta_{2 j}\right)$ of branch points. However, once the logarithmic branches $\log \left(z-z^{\prime}\right)$ are defined, the parameters $l_{i}$ as well as the prepotential $\mathcal{F}$ as functions of the 't Hooft parameters $\mathbf{s}$ are invariant under deformations of the cuts in fixed homology classes in $\mathbb{C} \backslash\left\{\beta_{i}\right\}_{i=1}^{2 q}$. To prove this property we follow a method used in 21]: let us take a fixed $\lambda \in \mathbb{C} \backslash \Gamma$, a fixed $z_{j} \in \gamma_{j}$ and two paths $\Gamma_{\lambda, \pm}$ in $\mathbb{C} \backslash \Gamma$ running from $z_{j \pm}$ to $\lambda$. Then since $y(z)=W^{\prime}(z)-2 g^{\prime}(z)$ for $z \in \mathbb{C} \backslash \Gamma$ and using (24) it follows that

$$
\int_{\Gamma_{\lambda,+}} y(z) \mathrm{d} z+\int_{\Gamma_{\lambda,-}} y(z) \mathrm{d} z=2\left(W(\lambda)-2 g(\lambda)-l_{j}\right) .
$$

Hence

$$
l_{j}=\lim _{\lambda \rightarrow \infty}\left(W(\lambda)-2 s \log \lambda-\frac{1}{2} \int_{\Gamma_{\lambda,+}} y(z) \mathrm{d} z-\frac{1}{2} \int_{\Gamma_{\lambda,-}} y(z) \mathrm{d} z\right) .
$$


Spectral curves in gauge/string dualities: integrability, singular sectors and regularization. 10

From this identity it is clear that the value of $l_{j}$ does not change if we deform the cuts in fixed homology classes in $\mathbb{C} \backslash\left\{\beta_{i}\right\}_{i=1}^{2 q}$ keeping the 't Hooft parameters constant. The same statement for the prepotential $\mathcal{F}$ follows at once taking into account that (15) and (24) imply

$$
\mathcal{F}=\frac{1}{2} \int_{\gamma} \rho(z) W(z)|\mathrm{d} z|+\frac{1}{2} \sum_{j=1}^{q} s_{j} l_{j}
$$

and that

$$
\int_{\gamma} \rho(z) W(z)|\mathrm{d} z|=-\frac{1}{4 \pi i} \sum_{j=1}^{q} \oint_{A_{j}} y(z) W(z) \mathrm{d} z
$$

\subsection{Integrable structure: Whitham equations}

Given a spectral curve (11) in the regular sector $\operatorname{reg} \mathcal{M}_{q}$ we may consider the genus $q-1$ hyperelliptic Riemann surface $M$ associated to the reduced curve

$$
y_{0}^{2}=\prod_{i=1}^{2 q}\left(z-\beta_{i}\right)
$$

which encodes the complete structure of the spectral curve. We are going to use the theory of Abelian differentials in Riemann surfaces (see Appendix A) to formulate a system of Whitham differential equations which characterize the cut endpoints as functions $\boldsymbol{\beta}(\mathbf{s})$ of the 't Hooft parameters. Furthermore, we will derive a system of implicit equations which determines the functions $\boldsymbol{\beta}(\mathbf{s})$ and exhibits the integrability of the Whitham differential equations.

Let $\left\{A_{i}, B_{i}\right\}_{i=1}^{q-1}$ be the homology basis of cycles in $M$ showed in figure 2 , where $A_{i}$ are counterclockwise cycles around the cuts $\gamma_{i}$ with endpoints $\left(\beta_{2 i-1}, \beta_{2 i}\right)$. We will denote the corresponding periods of a differential d $\omega$ in $M$ by

$$
A_{i}(\mathrm{~d} \omega)=\oint_{A_{i}} \mathrm{~d} \omega, \quad B_{i}(\mathrm{~d} \omega)=\oint_{B_{i}} \mathrm{~d} \omega
$$

Let us introduce the meromorphic differential

$$
\mathrm{d} \mathbb{S}=\frac{1}{2}\left(y(z)+W^{\prime}(z)\right) \mathrm{d} z
$$

where $\mathrm{y}(z)$ denotes the function on the Riemann surface $M$ determined by two branches of (1) given by

$$
y_{2}(z)=-y_{1}(z)=y(z) .
$$

We are going to decompose $d \mathbb{S}$ in terms of the set Abelian differentials of first kind (i.e., holomorphic) $\left\{\mathrm{d} \varphi_{i}\right\}_{i=1}^{q-1}$, second kind $\mathrm{d} \Omega_{n}(n \geq 1)$ and third kind $\mathrm{d} \Omega_{0}$ described in Appendix A. These Abelian differentials can be written as

$$
\mathrm{d} \varphi_{j}(z)=\frac{p_{j}(z)}{y_{0}(z)} \mathrm{d} z, \quad \mathrm{~d} \Omega_{n}=\left(\frac{n}{2} z^{n-1}+\frac{P_{n}(z)}{y_{0}(z)}\right) \mathrm{d} z, \quad \mathrm{~d} \Omega_{0}=\frac{P_{0}(z)}{y_{0}(z)} \mathrm{d} z,(37)
$$

for appropriate polynomials $p_{j}(z)$ and $P_{n}(z)$. 
Spectral curves in gauge/string dualities: integrability, singular sectors and regularization. 11

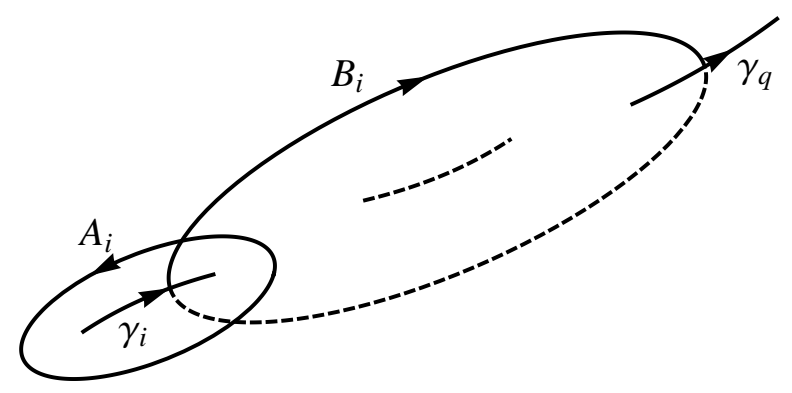

Figure 2. The homology basis.

We first observe that

$$
\mathrm{y}(z) \mathrm{d} z= \begin{cases}\left(W^{\prime}(z)-\frac{2 s}{z}+\mathcal{O}\left(z^{-2}\right)\right) \mathrm{d} z, & \text { as } z \rightarrow \infty_{1}, \\ \left(-W^{\prime}(z)+\frac{2 s}{z}+\mathcal{O}\left(z^{-2}\right)\right) \mathrm{d} z, & \text { as } z \rightarrow \infty_{2} .\end{cases}
$$

Since the only poles of $\mathrm{y}(z) \mathrm{d} z$ are at $\infty_{1}$ and $\infty_{2}$, from (38) we deduce that

$$
\mathrm{d} \mathbb{S}-\sum_{n=1}^{N+1} g_{n} \mathrm{~d} \Omega_{n}+s \mathrm{~d} \Omega_{0}
$$

is a first-kind Abelian differential in $M$. Consequently it admits a decomposition in the canonical basis

$$
\mathrm{d} \mathbb{S}-\sum_{n=1}^{N+1} g_{n} \mathrm{~d} \Omega_{n}+s \mathrm{~d} \Omega_{0}=\sum_{j=1}^{q-1} \lambda_{j} \mathrm{~d} \varphi_{j},
$$

for some complex coefficients $\lambda_{j} \in \mathbb{C}$. Thus, we may write

$$
\mathrm{y}(z) \mathrm{d} z=-W^{\prime}(z) \mathrm{d} z+2 \sum_{j=1}^{q-1} \lambda_{j} \mathrm{~d} \varphi_{j}+2 \sum_{n=1}^{N+1} g_{n} \mathrm{~d} \Omega_{n}-2 s \mathrm{~d} \Omega_{0} .
$$

Moreover, since $A_{i}\left(\mathrm{~d} \Omega_{n}\right)=0$ for all $i=1, \ldots, q-1$ and $n \geq 0$ then from (5) and (41) we get

$$
\lambda_{j}=-2 \pi \text { i } s_{j} .
$$

Therefore we obtain the decomposition

$$
\mathrm{d} \mathbb{S}=\sum_{n=1}^{N+1} g_{n} \mathrm{~d} \Omega_{n}-\sum_{j=1}^{q} s_{j}\left(\mathrm{~d} \Omega_{0}+2 \pi \mathrm{i}\left(1-\delta_{j q}\right) \mathrm{d} \varphi_{j}\right)
$$

The differential $d \mathbb{S}$ generates a system of Whitham equations with respect to the 't Hooft parameters $\mathbf{s}$ [23]- 24]. To derive this system we notice that $\mathrm{d} S$ has poles at $\infty_{1}$ and $\infty_{2}$ only. Moreover its derivatives with respect to the 't Hooft parameters satisfy

$$
\frac{\partial \mathrm{d} \mathbb{S}}{\partial s_{j}}=\left\{\begin{array}{l}
\left(-\frac{1}{z}+\mathcal{O}\left(z^{-2}\right)\right) \mathrm{d} z, \quad \text { as } z \rightarrow \infty_{1}, \\
\left(\frac{1}{z}+\mathcal{O}\left(z^{-2}\right)\right) \mathrm{d} z, \quad \text { as } z \rightarrow \infty_{2} .
\end{array}\right.
$$


Spectral curves in gauge/string dualities: integrability, singular sectors and regularization. 12

and

$$
A_{k}\left(\frac{\partial \mathrm{d} \mathbb{S}}{\partial s_{j}}\right)=-2 \pi \mathrm{i} \delta_{j k}, \quad k=1, \ldots, q-1,
$$

which imply

$$
\frac{\partial \mathrm{d} \mathbb{S}}{\partial s_{j}}=-\mathrm{d} \Omega_{0}-2 \pi \mathrm{i}\left(1-\delta_{j q}\right) \mathrm{d} \varphi_{j}
$$

As a consequence we have the following system of Whitham equations

$$
\frac{\partial}{\partial s_{i}}\left(\mathrm{~d} \Omega_{0}+2 \pi \mathrm{i}\left(1-\delta_{j q}\right) \mathrm{d} \varphi_{j}\right)=\frac{\partial}{\partial s_{j}}\left(\mathrm{~d} \Omega_{0}+2 \pi \mathrm{i}\left(1-\delta_{i q}\right) \mathrm{d} \varphi_{i}\right) .
$$

Using (37) we may rewrite (43) as

$$
\frac{\partial y(z)}{\partial s_{j}}=-2 \frac{P_{0}(z)-2 \pi \mathrm{i}\left(1-\delta_{j q}\right) p_{j}(z)}{y_{0}(z)} .
$$

Hence dividing both members of the resulting equations by $y_{0}(z)$ and equating the residues at $z=\beta_{i}$ we get the following system of ordinary differential equations

$$
\frac{\partial \beta_{i}}{\partial s_{j}}=4 \frac{P_{0}\left(\beta_{i}, \boldsymbol{\beta}\right)-2 \pi \mathrm{i}\left(1-\delta_{j q}\right) p_{j}\left(\beta_{i}, \boldsymbol{\beta}\right)}{\prod_{l=1}^{p}\left(\beta_{i}-\alpha_{l}\right) \prod_{k \neq i}\left(\beta_{i}-\beta_{k}\right)},
$$

which will be henceforth referred to as the Whitham equations for the branch points. From these equations it follows that for spectral curves in the regular sectors the branch points are analytic functions of the 't Hooft parameters.

From (41) and using (37), we have

$$
y(z) y_{0}(z)=2 \sum_{n=1}^{N+1} g_{n} P_{n}(z)-2 s P_{0}(z)-4 \pi \mathrm{i} \sum_{j=1}^{q-1} s_{j} p_{j}(z) .
$$

Therefore if we set $z=\beta_{i}$ in this identity we find the system

$$
\sum_{n=1}^{N+1} g_{n} P_{n}\left(\beta_{i}, \boldsymbol{\beta}\right)-\sum_{j=1}^{q} s_{j}\left(P_{0}\left(\beta_{i}, \boldsymbol{\beta}\right)+2 \pi \mathrm{i}\left(1-\delta_{j q}\right) p_{j}\left(\beta_{i}, \boldsymbol{\beta}\right)\right)=0,
$$

where $i=1, \ldots, 2 q$, which provides an implicit integration of the Whitham equations and determines the branch points in terms of the 't Hooft parameters. We observe that (48) means that the generating differential $d \mathbb{S}$ vanishes at the branch points $z=\beta_{i}$ [22].

\subsection{Prepotentials and $\tau$-functions}

As we have said before, the present work deals with the moduli space of spectral curves (1) for a fixed polynomial $W(z)$, but obviously, the coefficients $\mathbf{g}=\left(g_{1}, \ldots, g_{N}\right)$ of $W(z)$ can be also considered as free complex parameters too. In that case, repeating the analysis of the last subsection we immediately find

$$
\frac{\partial \mathrm{d} \mathbb{S}}{\partial g_{n}}=\mathrm{d} \Omega_{n}
$$

and consequently we may enlarge the systems of Whitham equations (44) and 46 including differential equations with respect to the parameters $\mathbf{g}$. In this sense it is 
Spectral curves in gauge/string dualities: integrability, singular sectors and regularization. 13

important to notice the properties of the prepotential functional as a function of these parameters. Indeed, from $(8)$ and $(24)$, taking into account that $\rho\left(\beta_{j}\right)=0$, we have

$$
\begin{aligned}
\frac{\partial \mathcal{F}}{\partial g_{n}} & =\int_{\gamma} z^{n} \rho(z)|\mathrm{d} z|+\int_{\gamma}\left(W(z)-\int_{\gamma} \log \left(z-z^{\prime}\right)^{2} \rho\left(z^{\prime}\right)\left|\mathrm{d} z^{\prime}\right|\right) \frac{\partial \rho(z)}{\partial g_{n}}|\mathrm{~d} z| \\
& =\int_{\gamma} z^{n} \rho(z)|\mathrm{d} z|+\int_{\gamma}\left(W(z)-\left(g\left(z_{+}\right)+g\left(z_{-}\right)\right)\right) \frac{\partial \rho(z)}{\partial g_{n}}|\mathrm{~d} z| \\
& =\int_{\gamma} z^{n} \rho(z)|\mathrm{d} z|+\sum_{j=1}^{q} l_{j} \int_{\gamma_{j}} \frac{\partial \rho(z)}{\partial g_{n}}|\mathrm{~d} z| \\
& =\int_{\gamma} z^{n} \rho(z)|\mathrm{d} z|+\sum_{j=1}^{q} l_{j} \frac{\partial s_{j}}{\partial g_{n}}=\int_{\gamma} z^{n} \rho(z)|\mathrm{d} z| .
\end{aligned}
$$

On the other hand from $(20)$ and $(35)$ it follows that as $z \rightarrow \infty_{1}$

$$
\mathrm{d} \mathbb{S}=\left(W^{\prime}(z)-\sum_{n \geq 1} \frac{1}{z^{n}} \int_{\gamma}\left(z^{\prime}\right)^{n-1} \rho\left(z^{\prime}\right)\left|\mathrm{d} z^{\prime}\right|\right) \mathrm{d} z .
$$

Then we conclude that

$$
\frac{\partial \mathcal{F}}{\partial g_{n}}=-\frac{1}{2 \pi \mathrm{i}} \oint_{\Gamma_{\infty}} z^{n} \mathrm{~d} \mathbb{S},
$$

where $\Gamma_{\infty}$ is a positively oriented circle of large radius.

From (24) and (36) we find that the parameters $l_{i}$ are related to the $B$-periods in the form

$$
\oint_{B_{i}} \mathrm{~d} \mathbb{S}=l_{q}-l_{i}, \quad i=1, \ldots, q-1,
$$

so that using 28 we have

$$
\frac{\partial \mathcal{F}}{\partial s_{q}}-\frac{\partial \mathcal{F}}{\partial s_{i}}=\oint_{B_{i}} \mathrm{~d} \mathbb{S}, \quad i=1, \ldots, q-1,
$$

The relations (49) and (51) mean that the logarithm $\log \mathcal{F}$ of the prepotential functional is a $\tau$-function of the Whitham hierarchy with respect to the variables $(\mathbf{g}, \mathbf{s})$.

\section{Spectral curves in terms of deformation parameters}

In the previous section the set $\mathbf{s}$ of 't Hooft parameters has been used to describe the integrable structure which underlies spectral curves. However, the use of the alternative set $\mathbf{t}$ of deformation parameters turns to be more convenient for dealing with several problems as, for example, the construction of explicit examples of multicut spectral curves.

\subsection{Roots of spectral curves as functions of deformation parameters}

The spectral curves in $\mathcal{M}_{q}$ satisfy the system of $2 N$ equations

$$
\left\{\begin{array}{cl}
P\left(\alpha_{l}, \mathbf{t}\right)=P^{\prime}\left(\alpha_{l}, \mathbf{t}\right)=0, & l=1, \ldots, p, \\
P\left(\beta_{i}, \mathbf{t}\right)=0, & i=1, \ldots, 2 q,
\end{array}\right.
$$


Spectral curves in gauge/string dualities: integrability, singular sectors and regularization. 14 where $P(z, \mathbf{t})$ denotes the polynomial

$$
P(z, \mathbf{t})=y(z, \mathbf{t})^{2}=W^{\prime}(z)^{2}+\sum_{k=1}^{N} t_{k} z^{N-k} .
$$

This system defines an affine variety in the space $\mathbb{C}^{2 N+q}$ of the variables $\left(\alpha_{l}, \beta_{i}, t_{k}\right)$. The Jacobian of the system $(52)$

$$
\frac{\partial\left(P\left(\alpha_{1}\right), \cdots, P\left(\alpha_{p}\right), P^{\prime}\left(\alpha_{1}\right), \cdots, P^{\prime}\left(\alpha_{p}\right), P\left(\beta_{1}\right), \cdots, P\left(\beta_{2 q}\right)\right)}{\partial\left(\alpha_{1}, \cdots, \alpha_{p}, \beta_{1}, \cdots, \beta_{2 q}, t_{1}, \cdots, t_{N}\right)}
$$

evaluated at solutions of $(52)$ is of the form

$$
\left(\begin{array}{c|c}
A & B \\
\hline C & D
\end{array}\right)
$$

where $A$ is the $p \times(p+2 q)$ zero matrix since from $(52)$

$$
A_{l m}=\delta_{l m} P^{\prime}\left(\alpha_{l}\right)=0, \quad l=1, \ldots, p, \quad m=1, \ldots, p+2 q .
$$

Furthermore $B$ is the Vandermonde matrix

$$
B=\left(\begin{array}{cccc}
\alpha_{1}^{N-1} & \alpha_{1}^{N-2} & \cdots & 1 \\
\alpha_{2}^{N-1} & \alpha_{2}^{N-2} & \cdots & 1 \\
\vdots & \vdots & \ddots & \vdots \\
\alpha_{p}^{N-1} & \alpha_{p}^{N-2} & \cdots & 1
\end{array}\right)
$$

$C$ is the diagonal matrix

$$
C=\operatorname{diag}\left(P^{\prime \prime}\left(\alpha_{1}\right), \cdots, P^{\prime \prime}\left(\alpha_{p}\right), P^{\prime}\left(\beta_{1}\right), \cdots, P^{\prime}\left(\beta_{2 q}\right)\right)
$$

and

$$
D=\left(\begin{array}{cccc}
(N-1) \alpha_{1}^{N-2} & (N-2) \alpha_{1}^{N-3} & \cdots & 0 \\
(N-1) \alpha_{2}^{N-1} & (N-2) \alpha_{2}^{N-3} & \cdots & 0 \\
\vdots & \vdots & \ddots & \vdots \\
(N-1) \alpha_{p}^{N-2} & (N-2) \alpha_{p}^{N-3} & \cdots & 0 \\
\beta_{1}^{N-1} & \beta_{1}^{N-2} & \cdots & 1 \\
\beta_{2}^{N-1} & \beta_{2}^{N-2} & \cdots & 1 \\
\vdots & \vdots & \ddots & \vdots \\
\beta_{2 q}^{N-1} & \beta_{2 q}^{N-2} & \cdots & 1
\end{array}\right)
$$

Let us consider now spectral curves in the regular sector $\operatorname{reg} \mathcal{M}_{q}$. It is easy to see that for any set of $q$ deformation parameters $\left(t_{i_{1}}, \cdots, t_{i_{q}}\right)$ the minor of the Jacobian corresponding to the derivatives with respect to the variables $\left(\alpha_{l}, \beta_{i}\right)$ and the remaining $N-q$ deformation parameters $t_{k}\left(k \notin\left\{i_{1}, \ldots, i_{q}\right\}\right)$ is a non singular $(2 N) \times(2 N)$ matrix. Therefore the implicit function Theorem implies

Theorem 1 For spectral curves in the regular sector $\operatorname{reg} \mathcal{M}_{q}$, each set of $q$ different deformation parameters $\left(t_{i_{1}}, \cdots, t_{i_{q}}\right)$ defines an analytic system of coordinates, i.e. all the variables $\left(\alpha_{l}, \beta_{i}, t_{k}\right)$ are local analytic functions of $\left(t_{i_{1}}, \cdots, t_{i_{q}}\right)$ 
Spectral curves in gauge/string dualities: integrability, singular sectors and regularization. 15

If we eliminate the variables $\left(\alpha_{l}, \beta_{i}\right)$ in the system (52) or, alternatively, in the system obtained identifying coefficients of powers of $z$ between (1) and (11), we get a set of $N-q$ constraints

$$
f_{k}(\mathbf{t})=0, \quad k=1, \ldots, N-q,
$$

which characterizes the class $\mathcal{M}_{q}$ in the space $\mathbb{C}^{N}$ of deformation parameters. These constraints can be used to define analytic systems of coordinates in reg $\mathcal{M}_{q}$ given by sets $\left(t_{i_{1}}, \cdots, t_{i_{q}}\right)$ of $q$ distinct deformation parameters.

The class $\mathcal{M}_{N}$ contains all the spectral curves (1) and will be called the generic class. Its elements can be factorized in the generic form

$$
y(z, \mathbf{t})^{2}=\prod_{j=1}^{2 N}\left(z-\beta_{j}\right),
$$

where the roots $\beta_{i}$ may coincide. In the case of the generic class $\mathcal{M}_{N}$ the set of the deformation parameters $\left(t_{1}, \ldots, t_{N}\right)$ defines an analytic system of coordinates in the regular sector reg $\mathcal{M}_{N}$. Obviously the domain in the space of deformation parameters $\mathbb{C}^{N}$ which corresponds to reg $\mathcal{M}_{N}$ is the open set determined by

$$
D(\mathbf{t}) \neq 0,
$$

where $D=D(\mathbf{t})$ is the discriminant of the polynomial $y(z, \mathbf{t})^{2}$. Consequently $\operatorname{sing} \mathcal{M}_{N}$ is represented by the hypersurface determined by the constraint

$$
D(\mathbf{t})=0 .
$$

From the point of view of the applications to gauge/string dualities it is important to determine under what conditions a map $\left(t_{i_{1}}, \cdots, t_{i_{q}}\right) \mapsto\left(s_{1}, \ldots, s_{q}\right)$ relating the coordinate charts of deformation parameters and 't Hooft parameters defines an analytic change of variables. Now from (5) we conclude that the Jacobian matrix of these transformation is given by

$$
\frac{\partial s_{j}}{\partial t_{i_{k}}}=-\frac{1}{8 \pi \mathrm{i}} \oint_{A_{j}}\left(z^{N-i_{k}}+\sum_{i \notin\left\{i_{1}, \ldots, i_{q}\right\}} z^{N-i} \frac{\partial t_{i}}{\partial t_{i_{k}}}\right) \frac{\mathrm{d} z}{y(z)} .
$$

Thus the question reduces to the verification of the nonsingularity of this Jacobian matrix. For spectral curves in $\operatorname{reg} \mathcal{M}_{N}$ it follows from (5) that

$$
\frac{\partial s_{j}}{\partial t_{k}}=-\frac{1}{8 \pi \mathrm{i}} \oint_{A_{j}} \frac{z^{N-k}}{\sqrt{\prod_{j=1}^{2 N}\left(z-\beta_{j}\right)}} \mathrm{d} z, \quad j, k=2 \ldots, N,
$$

This means that the Jacobian of the transformation $\left(t_{2}, \ldots, t_{N}\right) \mapsto\left(s_{2}, \ldots, s_{N}\right)$ is non singular since it is the matrix of $A$-periods of a basis of Abelian differentials in the Riemann surface given by

$$
y(z)^{2}=\prod_{j=1}^{2 N}\left(z-\beta_{j}\right),
$$

where all the branch points are different ( $N-1$ genus). Moreover, according to (21) it follows at once that $t_{1}=-4 s$, then the change of variables $\left(t_{1}, \ldots, t_{N}\right) \mapsto\left(s_{1}, \ldots, s_{N}\right)$ 
Spectral curves in gauge/string dualities: integrability, singular sectors and regularization. 16

is an analytic diffeomorphism locally around any point in the regular sector $\operatorname{reg} \mathcal{M}_{q}$. Consequently using Theorem 1 we conclude that the set of 't Hooft parameters $\left(s_{1}, \ldots, s_{N}\right)$ provides an analytic system of coordinates in $\operatorname{reg} \mathcal{M}_{N}$.

\subsection{Whitham equations with respect to deformation parameters}

We have seen that for spectral curves in the regular sector $\operatorname{reg} \mathcal{M}_{q}$ all the variables $\left(\alpha_{l}, \beta_{i}, t_{k}\right)$ can be expressed as analytic functions in any coordinate system of $q$ deformation parameters. In particular one may consider the expressions for the simple roots in terms of the coordinates $\left(t_{1}, \ldots, t_{q}\right)$

$$
\beta_{i}=\beta_{i}\left(t_{1}, t_{2}, \ldots, t_{q}\right) .
$$

We are going to derive the system of Whitham equations which characterizes the functions (60). Indeed, taking into account that the double roots $\alpha_{l}$ are distinct then from (53) we get the identities

$$
t_{l+q}=\sum_{m=1}^{p} V_{l m}(\boldsymbol{\alpha})\left[P\left(\alpha_{m}, \mathbf{t}\right)-\left(W^{\prime}\left(\alpha_{m}\right)^{2}+\sum_{j=1}^{q} t_{j} \alpha_{m}^{N-j}\right)\right],
$$

for $l=1, \ldots, p=N-q$ where $V_{l m}(\boldsymbol{\alpha})$ is the inverse of the Vandermonde matrix $\left(\alpha_{l}^{p-m}\right)_{l, m=1}^{p}$. As a consequence the functions

$$
P\left(\beta_{i}, \mathbf{t}\right)-\sum_{l, m=1}^{p} \beta_{i}^{p-l} V_{l m}(\boldsymbol{\alpha}) P\left(\alpha_{m}, \mathbf{t}\right)
$$

do not depend on the deformation parameters $\left(t_{q+1}, \ldots, t_{N}\right)$ since they can be written as

$$
Q\left(\boldsymbol{\alpha}, \beta_{i}\right)+\sum_{j=1}^{q} t_{j}\left(\beta_{i}^{N-j}-\sum_{l, m=1}^{p} \beta_{i}^{p-l} V_{l m}(\boldsymbol{\alpha}) \alpha_{m}^{N-j}\right),
$$

where

$$
Q(\boldsymbol{\alpha}, \beta)=W^{\prime}(\beta)^{2}-\sum_{l, m=1}^{p} \beta^{p-l} V_{l m}(\boldsymbol{\alpha}) W^{\prime}\left(\alpha_{m}\right)^{2} .
$$

Now for spectral curves in reg $\mathcal{M}_{q}$ we may express all the variables $\left(\alpha_{l}, \beta_{i}, t_{k}\right)$ as functions of the coordinates $\left(t_{1}, \ldots, t_{q}\right)$. Hence differentiating the equations

$$
P\left(\beta_{i}, \mathbf{t}\right)-\sum_{l, m} \beta_{i}^{p-l} V_{l m}(\boldsymbol{\alpha}) P\left(\alpha_{m}, \mathbf{t}\right)=0
$$

with respect to the free parameters $\left\{t_{j}\right\}_{j=1}^{q}$, taking into account that $P\left(\alpha_{l}, \mathbf{t}\right)=$ $P^{\prime}\left(\alpha_{l}, \mathbf{t}\right)=0$, we obtain the system of differential equations

$$
\frac{\partial \beta_{i}}{\partial t_{j}}=\frac{\sum_{l, m} \beta_{i}^{p-l} V_{l m}(\boldsymbol{\alpha}) \alpha_{m}^{N-j}-\beta_{i}^{N-j}}{\prod_{l}\left(\alpha_{l}-\beta_{i}\right)^{2} \prod_{j \neq i}\left(\beta_{i}-\beta_{j}\right)} .
$$

This is the system of Whitham equations for the branch points with respect to the deformation parameters. 
Spectral curves in gauge/string dualities: integrability, singular sectors and regularization. 17

The system (63) shows how the functions $\beta_{i}\left(t_{1}, \ldots, t_{q}\right)$ become singular for spectral curves in the singular sector $\operatorname{sing} \mathcal{M}_{q}$, where at least two roots of the set $\left(\alpha_{l}, \beta_{i}\right)$ coalesce.

For $q=N$ and $q=N-1$ the system reduces to

$$
\frac{\partial \beta_{i}}{\partial t_{j}}=-\frac{\beta_{i}^{N-j}}{\prod_{j \neq i}\left(\beta_{i}-\beta_{j}\right)},
$$

and

$$
\frac{\partial \beta_{i}}{\partial t_{j}}=\frac{\alpha^{N-j}-\beta_{i}^{N-j}}{\left(\alpha-\beta_{i}\right)^{2} \prod_{j \neq i}\left(\beta_{i}-\beta_{j}\right)}, \quad\left(\alpha=-N g_{N}-\frac{\beta_{1}+\cdots+\beta_{2 q}}{2}\right)
$$

respectively.

\subsection{The Gaussian and cubic models}

A complete explicit analysis of the classes $\mathcal{M}_{q}$ is possible only for polynomials $W(z)$ of low degree. We next discuss some examples for the cases of degrees two and three. Similar studies have been recently performed for the cubic model in 28] and for the quartic model in [29].

Gaussian model For the Gaussian model

$$
W(z)=\frac{z^{2}}{2}
$$

we have $N=1$ and $y(z, \mathbf{t})^{2}=z^{2}-4 s$. The system (52) for $q=1$ and $q=0$ reduce to

$$
\beta_{i}^{2}-4 s=0, \quad i=1,2 \text {, }
$$

and

$$
\alpha^{2}-4 s=0, \quad \alpha=0,
$$

respectively. Thus for $\mathcal{M}_{1}$ the branchpoints are

$$
\beta_{1}=-\beta_{2}=2 \sqrt{s} \text {. }
$$

The regular sector reg $\mathcal{M}_{1}$ is given by the spectral curves corresponding to values $s \neq 0$. The singular sector sing $\mathcal{M}_{1}$ coincides with $\mathcal{M}_{0}$ and contains only the spectral curve $y(z)^{2}=z^{2}$ which corresponds to $s=0$.

Cubic model Let us consider the cubic model

$$
W(z)=\frac{z^{3}}{3}-g z, \quad g \in \mathbb{C} \backslash\{0\} .
$$

In this case $N=2$ and

$$
y(z, \mathbf{t})^{2}=\left(z^{2}-g\right)^{2}+t_{1} z+t_{2}, \quad t_{1}=-4 s .
$$

The generic class $\mathcal{M}_{2}$ is the set of all the spectral curves (69). If we write

$$
y(z, \mathbf{t})^{2}=\left(z-\beta_{1}\right)\left(z-\beta_{2}\right)\left(z-\beta_{3}\right)\left(z-\beta_{4}\right),
$$


Spectral curves in gauge/string dualities: integrability, singular sectors and regularization. 18 introduce the variables

$$
\begin{aligned}
& u_{1}=\frac{\beta_{1}+\beta_{2}}{2}, \quad u_{2}=\frac{\beta_{3}+\beta_{4}}{2}, \\
& \delta_{1}=\frac{\beta_{1}-\beta_{2}}{2}, \quad \delta_{2}=\frac{\beta_{3}-\beta_{4}}{2} .
\end{aligned}
$$

and identify the coefficients of powers of $z$ in equations 69 and $(70$ we obtain the relations

$$
u_{2}=-u_{1}, \quad \delta_{1}^{2}=g-u_{1}^{2}+\frac{s}{u_{1}}, \quad \delta_{2}^{2}=g-u_{1}^{2}-\frac{s}{u_{1}},
$$

and the equation which determines $u_{1}$ as a function of the deformation parameters $\left(s, t_{2}\right)$

$$
4 u_{1}^{6}-4 g u_{1}^{4}-t_{2} u_{1}^{2}-s^{2}=0 .
$$

The regular sector $\operatorname{reg} \mathcal{M}_{2}$ is determined by the points $\left(s, t_{2}\right) \in \mathbb{C}^{2}$ at which the discriminant of $y(z, \mathbf{t})^{2}$ does not vanish, i.e.

$$
\operatorname{reg} \mathcal{M}_{2} \leftrightarrow\left\{\left(s, t_{2}\right) \in \mathbb{C}^{2}: 27 s^{4}+\left(18 g t_{2}+16 g^{3}\right) s^{2}-t_{2}^{3}-g^{2} t_{2}^{2} \neq 0\right\} .
$$

The singular sector $\operatorname{sing} \mathcal{M}_{2}$ is given by the spectral curves 69 which have a multiple root. Their possible sets of root multiplicities are

$$
\left[2_{1}, 1_{2}\right],\left[1_{1}, 1_{3}\right],\left[2_{2}\right],\left[1_{4}\right],
$$

which obviously means that

$$
\operatorname{sing} \mathcal{M}_{2}=\mathcal{M}_{1}
$$

Let us now consider the class $\mathcal{M}_{1}$

$$
y(z, \mathbf{t})^{2}=(z-\alpha)^{2}\left(z-\beta_{1}\right)\left(z-\beta_{2}\right) .
$$

Equating (69) and (75), identifying coefficients and using the variables

$$
u=\frac{\beta_{1}+\beta_{2}}{2}, \quad \delta=\frac{\beta_{1}-\beta_{2}}{2},
$$

we get the system of equations

$$
\left\{\begin{array}{c}
\alpha=-u, \quad 2 u^{2}+\delta^{2}=2 g \\
u \delta^{2}=2 s, \quad u^{4}-u^{2} \delta^{2}-g^{2}=t_{2} .
\end{array}\right.
$$

Therefore $u$ satisfies the cubic equation

$$
u^{3}-g u+s=0
$$

and $\delta^{2}$ is determined by

$$
\begin{array}{ll}
\delta^{2}=\frac{2 s}{u}, & \text { if } u \neq 0, \\
\delta^{2}=2 g, & \text { if } u=0 .
\end{array}
$$

Moreover, eliminating $u$ and $\delta^{2}$ in 76 we obtain a constraint for the deformation parameters $\left(s, t_{2}\right)$ which, as it should be expected, is equivalent to the vanishing of the discriminant of $y(z, \mathbf{t})^{2}$

$$
\mathcal{M}_{1} \leftrightarrow\left\{\left(s, t_{2}\right) \in \mathbb{C}^{2}: 27 s^{4}+\left(18 g t_{2}+16 g^{3}\right) s^{2}-t_{2}^{3}-g^{2} t_{2}^{2}=0\right\} .
$$


Spectral curves in gauge/string dualities: integrability, singular sectors and regularization. 19

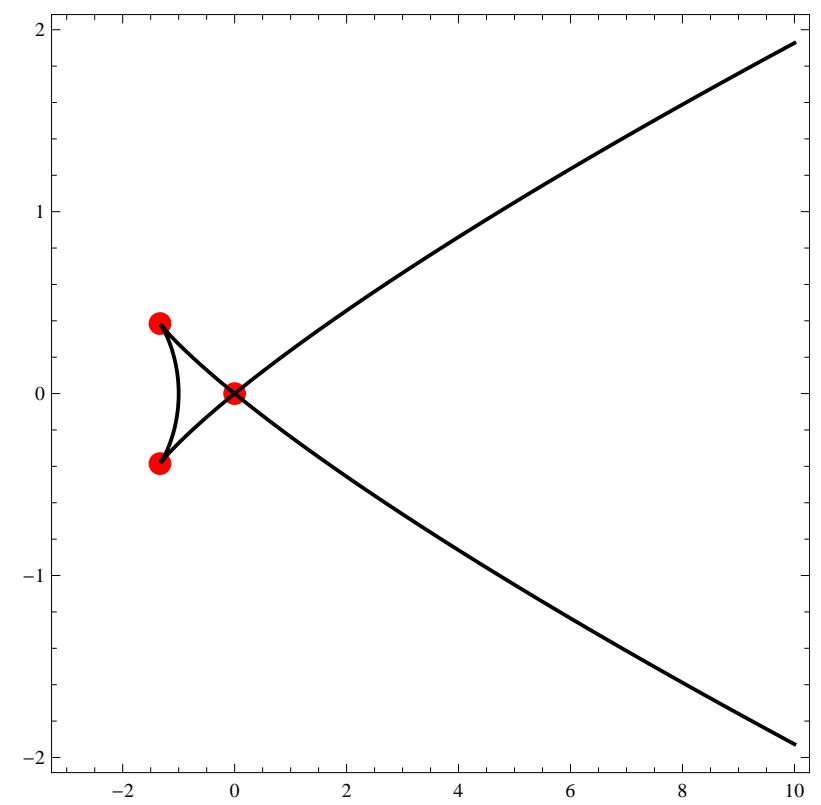

Figure 3. The classes of spectral curves of the cubic model for $g=1$ in the space of real values of $\left(t_{2}, s\right)$. The whole real plane describes $\mathcal{M}_{2}$, the curve corresponds to the implicit equation $(80)$ and represents $\operatorname{sing} \mathcal{M}_{2}=\mathcal{M}_{1}$, the three marked points represent $\operatorname{sing} \mathcal{M}_{1}$. The point $(0,0)$ represents $\mathcal{M}_{0}$.

According to 76$) \beta_{1}=\beta_{2}$ if and only if $\left(s, t_{2}\right)=(0,0)$, i.e. $y^{2}=\left(z^{2}-g\right)^{2}$. Moreover if $\alpha$ coincides with $\beta_{1}$ or $\beta_{2}$ then the polynomial $y(z)^{2}$ acquires a root of multiplicity $m \geq 3$ and therefore it satisfies 30

$$
\sum_{k=0}^{4}(-1)^{k} D_{z}^{k} y^{2}(z) D_{z}^{4-k} y^{2}(z)=0,
$$

which gives the constraint

$$
3 t_{2}+4 g^{2}=0,
$$

or, due to 80 equivalently,

$$
\left(s, t_{2}\right)=\left( \pm 2\left(\frac{g}{3}\right)^{3 / 2},-\frac{4}{3} g^{2}\right) .
$$

Therefore, the singular sector $\operatorname{sing} \mathcal{M}_{1}$ is given by only three points of the space of deformation parameters

$$
\operatorname{sing} \mathcal{M}_{1} \leftrightarrow\left\{\left(s, t_{2}\right)=(0,0),\left( \pm 2\left(\frac{g}{3}\right)^{3 / 2},-\frac{4}{3} g^{2}\right)\right\} .
$$

The corresponding root multiplicities are $\left[2_{2}\right]$ for $(0,0)$ and $\left[1_{1}, 1_{3}\right]$ for $\left( \pm 2(g / 3)^{3 / 2},-4 g^{2} / 3\right)$. Figure 4 exhibits an example of a curve in $\operatorname{sing} \mathcal{M}_{1}$ in which a triple root is formed as the outcome of a merging of a simple root and the double root.

From (77) it follows that the class $\mathcal{M}_{1}$ decomposes into three subsets

$$
\mathcal{M}_{1}=\cup_{k=0}^{2} \mathcal{M}_{1}^{(k)}
$$


Spectral curves in gauge/string dualities: integrability, singular sectors and regularization. 20

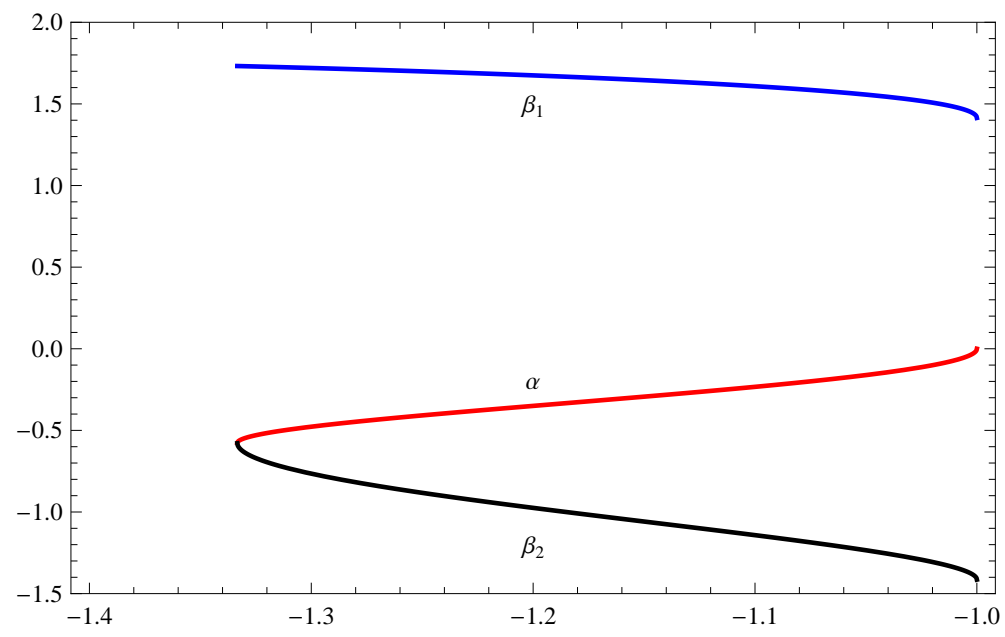

Figure 4. The functions $\alpha\left(t_{2}\right)$ and $\beta_{i}\left(t_{2}\right)$ at a merging of the double root and a simple root in the cubic model for $g=1$.

which are determined by the three branches

$$
u_{k}(s)=-\frac{g}{3 \Delta_{k}(s)}-\Delta_{k}(s), \quad(k=0,1,2)
$$

where

$$
\Delta_{k}(s)=\mathrm{e}^{\mathrm{i} 2 \pi k / 3} \sqrt[3]{\frac{s}{2}+\sqrt{\frac{s^{2}}{4}-\left(\frac{g}{3}\right)^{3}}} .
$$

The expansions of $u_{k}(s)$ as $s \rightarrow 0$ are

$$
\begin{aligned}
& u_{0}(s)=-\sqrt{g}-\frac{s}{2 g}+\frac{3 s^{2}}{8 g^{5 / 2}}+\mathcal{O}\left(s^{3}\right), \\
& u_{1}(s)=\sqrt{g}-\frac{s}{2 g}-\frac{3 s^{2}}{8 g^{5 / 2}}+\mathcal{O}\left(s^{3}\right), \\
& u_{2}(s)=\frac{s}{g}+\mathcal{O}\left(s^{3}\right),
\end{aligned}
$$

which show that in this limit the branches $u_{0}$ and $u_{1}$ represent families of one-cut spectral curves in which the cuts shrink to the corresponding critical point $-\sqrt{g}$ and $+\sqrt{g}$ of $W(z)$.

The solution $u_{2}(s)$ has a different behavior as $s \rightarrow 0$, because in this limit $u_{2} \rightarrow 0$, $\delta^{2} \rightarrow 2 g$ and therefore the branch points $\beta_{1}$ and $\beta_{2}$ reduce to two different points $\sqrt{2 g}$ and $-\sqrt{2 g}$. Note also from (76) that $t_{2} \rightarrow-g^{2}$ as $s \rightarrow 0$.

Finally, the class $\mathcal{M}_{0}$ contains only one spectral curve $y^{2}=\left(z^{2}-g\right)^{2}$ which corresponds to $\left(s, t_{2}\right)=(0,0)$.

\section{The Euler-Poisson-Darboux equation and the reduced systems}

As we have seen the regular sectors $\operatorname{reg} \mathcal{M}_{q}$ are completely characterized by the functions (60). We will now prove that these functions can be determined from a system of $2 q$ 
Spectral curves in gauge/string dualities: integrability, singular sectors and regularization. 21 equations for the variables

$$
\boldsymbol{\beta}=\left(\beta_{1}, \ldots, \beta_{2 q}\right), \quad \mathbf{t}^{(q)}=\left(t_{1}, \ldots, t_{q}\right)
$$

These systems will be called reduced systems. They are determined from a particular polynomial solution of the Euler-Poisson-Darboux equation $E\left(-\frac{1}{2},-\frac{1}{2}\right)$ (see e.g. [25])

$$
2\left(\beta_{j}-\beta_{i}\right) \frac{\partial^{2} \mathbb{W}}{\partial \beta_{i} \partial \beta_{j}}=\frac{\partial \mathbb{W}}{\partial \beta_{i}}-\frac{\partial \mathbb{W}}{\partial \beta_{j}} .
$$

\subsection{Reduced systems}

Let us define the function

$$
\mathbb{W}(\boldsymbol{\beta}, \mathbf{t})=\oint_{\Gamma_{\infty}} y_{0}(z, \boldsymbol{\beta}) y(z, \mathbf{t}) \frac{\mathrm{d} z}{2 \pi \mathrm{i}},
$$

where

$$
y_{0}(z, \boldsymbol{\beta})=\sqrt{\prod_{i=1}^{2 q}\left(z-\beta_{i}\right)}, \quad y(z, \mathbf{t})=\sqrt{W^{\prime}(z)^{2}+f(z, \mathbf{t})} .
$$

and $\Gamma_{\infty}$ is a positively oriented circle of large radius. It obviously satisfies the EulerPoisson-Darboux equation (89). Moreover, using the expansion of $y(z, \mathbf{t})$ at $z \rightarrow \infty$, i.e.

$$
y(z, \mathbf{t})=\sqrt{W^{\prime}(z)^{2}+f(z, \mathbf{t})}=W^{\prime}(z)+\frac{1}{2} \frac{f(z, \mathbf{t})}{W^{\prime}(z)}+\mathcal{O}\left(\frac{1}{z^{N+2}}\right),
$$

we deduce that $\mathbb{W}$ reduces to

$$
\begin{aligned}
& \mathbb{W}(\boldsymbol{\beta}, \mathbf{t})=\oint_{\Gamma_{\infty}} W^{\prime}(z) y_{0}(z, \boldsymbol{\beta}) \frac{\mathrm{d} z}{2 \pi \mathrm{i}} \\
& +\frac{1}{2} \sum_{j=1}^{q} t_{j} \oint_{\Gamma_{\infty}} z^{N-j} \frac{y_{0}(z, \boldsymbol{\beta})}{W^{\prime}(z)} \frac{\mathrm{d} z}{2 \pi \mathrm{i}}+\frac{t_{q+1}}{2} .
\end{aligned}
$$

Observe that $\mathbb{W}-t_{q+1} / 2$ does not depend on $\left(t_{q+1}, \ldots, t_{N}\right)$ and that it is linear in $\left(t_{1}, \ldots, t_{q}\right)$.

Examples: In the cubic model we have for $q=1$

$$
\mathbb{W}=\frac{1}{128}\left[-\left(\beta_{1}-\beta_{2}\right)^{2}\left(5 \beta_{1}^{2}+6 \beta_{2} \beta_{1}+5 \beta_{2}^{2}-16 g\right)-32\left(\beta_{1}+\beta_{2}\right) t_{1}+64 t_{2}\right]
$$

and for $q=2$

$$
\begin{aligned}
& \mathbb{W}=\frac{1}{256}\left[2\left(\beta_{1}+\beta_{2}+\beta_{3}+\beta_{4}\right)\left(\beta_{1}-\beta_{2}\right)^{2}\left(\beta_{3}-\beta_{4}\right)^{2}\right. \\
& +\left(\beta_{1}-\beta_{2}\right)^{2}\left(-7 \beta_{1}^{3}-9 \beta_{2} \beta_{1}^{2}+5 \beta_{3} \beta_{1}^{2}+5 \beta_{4} \beta_{1}^{2}-9 \beta_{2}^{2} \beta_{1}\right. \\
& \left.+6 \beta_{2} \beta_{3} \beta_{1}+6 \beta_{2} \beta_{4} \beta_{1}-7 \beta_{2}^{3}+5 \beta_{2}^{2} \beta_{3}+5 \beta_{2}^{2} \beta_{4}\right) \\
& +\left(\beta_{3}-\beta_{4}\right)^{2}\left(-7 \beta_{3}^{3}+5 \beta_{1} \beta_{3}^{2}+5 \beta_{2} \beta_{3}^{2}-9 \beta_{4} \beta_{3}^{2}-9 \beta_{4}^{2} \beta_{3}\right.
\end{aligned}
$$


Spectral curves in gauge/string dualities: integrability, singular sectors and regularization. 22

$$
\begin{aligned}
& \left.\left.+6 \beta_{1} \beta_{4} \beta_{3}+6 \beta_{2} \beta_{4} \beta_{3}-7 \beta_{4}^{3}+5 \beta_{1} \beta_{4}^{2}+5 \beta_{2} \beta_{4}^{2}\right)\right] \\
& +\frac{1}{16}\left(\beta_{1}+\beta_{2}-\beta_{3}-\beta_{4}\right)\left(\beta_{1}-\beta_{2}+\beta_{3}-\beta_{4}\right)\left(\beta_{1}-\beta_{2}-\beta_{3}+\beta_{4}\right) g \\
& +\frac{1}{16}\left[-\left(\beta_{1}-\beta_{2}+\beta_{3}-\beta_{4}\right)^{2}+4\left(\beta_{1} \beta_{3}+\beta_{2} \beta_{4}\right)+8 g\right] t_{1} \\
& -\frac{1}{4}\left(\beta_{1}+\beta_{2}+\beta_{3}+\beta_{4}\right) t_{2}
\end{aligned}
$$

We are now ready to prove the following characterization of the functions (60)

Theorem 2 For spectral curves in $\mathcal{M}_{q}$ the functions $\boldsymbol{\beta}=\boldsymbol{\beta}\left(\mathbf{t}^{(q)}\right)$ satisfy

$$
\frac{\partial \mathbb{W}}{\partial \beta_{i}}=0 \text { at } \boldsymbol{\beta}=\boldsymbol{\beta}\left(\mathbf{t}^{(q)}\right), \quad i=1, \ldots, 2 q .
$$

Proof One has

so

$$
\frac{\partial y_{0}(z, \boldsymbol{\beta})}{\partial \beta_{i}}=-\frac{1}{2} \frac{y_{0}(z, \boldsymbol{\beta})}{z-\beta_{i}}
$$

$$
\frac{\partial \mathbb{W}}{\partial \beta_{i}}=-\frac{1}{2} \oint_{\Gamma_{\infty}} \frac{y_{0}(z, \boldsymbol{\beta}) y(z, \mathbf{t})}{z-\beta_{i}} \frac{\mathrm{d} z}{2 \pi \mathrm{i}} .
$$

Moreover for spectral curves in $\mathcal{M}_{q}$ we have that

$$
\frac{y_{0}(z, \boldsymbol{\beta}) y(z, \mathbf{t})}{z-\beta_{i}}=\prod_{l=1}^{p}\left(z-\alpha_{l}\right) \prod_{j \neq i}\left(z-\beta_{j}\right)
$$

and then the statement follows.

The equations (92) can be expressed as

$$
\oint_{\Gamma_{\infty}} \frac{\prod_{j \neq i}\left(z-\beta_{j}\right)}{y_{0}(z, \boldsymbol{\beta})}\left(W^{\prime}(z)+\frac{1}{2} \frac{\sum_{k=1}^{q} t_{k} z^{N-k}}{W^{\prime}(z)}\right) \frac{\mathrm{d} z}{2 \pi \mathrm{i}}=0, \quad i=1, \ldots 2 q,
$$

and therefore for distinct $\beta_{i}$ they are equivalent to the system

$$
\left\{\begin{array}{l}
\oint_{\Gamma_{\infty}} \frac{z^{k}}{\sqrt{\prod_{j=1}^{2 q}\left(z-\beta_{j}\right)}}\left(W^{\prime}(z)+\frac{1}{2} \frac{\sum_{j=1}^{q} t_{j} z^{N-j}}{W^{\prime}(z)}\right) \frac{\mathrm{d} z}{2 \pi \mathrm{i}}=0, \\
k=0, \ldots 2 q-1
\end{array}\right.
$$

We will refer to 92 as the reduced system for the $3 q$ variables $\left(\boldsymbol{\beta}, \mathbf{t}^{(q)}\right)$. It must be observed that the previous system (52) for $\left(\alpha_{l}, \beta_{i}, t_{k}\right)$ involves a larger number $2 p+3 q$ of variables .

Examples: For $q=1$ the reduced system $(92)$ is the well-known pair of equations which determines the planar limit of random matrix models 26]

$$
\left\{\begin{array}{c}
\oint_{\Gamma_{\infty}} \frac{W^{\prime}(z)}{\sqrt{\left(z-\beta_{1}\right)\left(z-\beta_{2}\right)}} \mathrm{d} z=0 \\
\oint_{\Gamma_{\infty}} z \frac{W^{\prime}(z)}{\sqrt{\left(z-\beta_{1}\right)\left(z-\beta_{2}\right)}} \mathrm{d} z=4 \pi \mathrm{i} s .
\end{array}\right.
$$


Spectral curves in gauge/string dualities: integrability, singular sectors and regularization. 23

For $q=2$ the reduced system 92 takes the form

$$
\left\{\begin{array}{l}
\oint_{\Gamma_{\infty}} \frac{W^{\prime}(z)}{\sqrt{\prod_{j=1}^{4}\left(z-\beta_{j}\right)}} \mathrm{d} z=0, \\
\oint_{\Gamma_{\infty}} z \frac{W^{\prime}(z)}{\sqrt{\prod_{j=1}^{4}\left(z-\beta_{j}\right)}} \mathrm{d} z=0, \\
\oint_{\Gamma_{\infty}} z^{2} \frac{W^{\prime}(z)}{\sqrt{\prod_{j=1}^{4}\left(z-\beta_{j}\right)}} \mathrm{d} z=4 \pi \mathrm{i} s, \\
\oint_{\Gamma_{\infty}} z^{3} \frac{W^{\prime}(z)}{\sqrt{\prod_{j=1}^{4}\left(z-\beta_{j}\right)}} \mathrm{d} z=4 \pi \mathrm{i}\left(\frac{1}{2} \sum_{j=1}^{4} \beta_{j}-N g_{N}\right) s-\pi \mathrm{i} t_{2} .
\end{array}\right.
$$

\subsection{The classes $\mathcal{R}_{q}$ of solutions and their singular sectors}

The reduced systems allow us to establish a simple characterization of relevant subsets of singular spectral curves.

Definition 3 We define $\mathcal{R}_{q}$ as the set of all the solutions $\left(\boldsymbol{\beta}, \mathbf{t}^{(q)}\right)$ of the reduced system 92) such that $\beta_{i} \neq \beta_{j}$ for all $i \neq j$

Obviously for spectral curves in the regular sector $\operatorname{reg} \mathcal{M}_{q}$ we have that $\left(\boldsymbol{\beta}\left(\mathbf{t}^{(q)}\right), \mathbf{t}^{(q)}\right) \in$ $\mathcal{R}_{q}$. Nevertheless, we are interested in degenerate solutions $\left(\boldsymbol{\beta}, \mathbf{t}^{(q)}\right) \in \mathcal{R}_{q}$ at which the Jacobian of the system (92) vanishes.

$$
\left|\frac{\partial^{2} \mathbb{W}(\boldsymbol{\beta}, \mathbf{t})}{\partial \beta_{i} \partial \beta_{j}}\right|=0 .
$$

From the Euler-Poisson-Darboux equations $(89)$ it follows that if $\left(\boldsymbol{\beta}, \mathbf{t}^{(q)}\right) \in \mathcal{R}_{q}$ then all the derivatives of $\mathbb{W}$ at $\left(\boldsymbol{\beta}, \mathbf{t}^{(q)}\right)$ can be expressed in terms of linear combinations of derivatives of the form $\partial_{\beta_{i}}^{k} \mathbb{W}$. In particular if $\left(\boldsymbol{\beta}, \mathbf{t}^{(q)}\right) \in \mathcal{R}_{q}$ we have

$$
\left|\frac{\partial^{2} \mathbb{W}(\boldsymbol{\beta}, \mathbf{t})}{\partial \beta_{i} \partial \beta_{j}}\right|=\prod_{i=1}^{2 q} \frac{\partial^{2} \mathbb{W}(\boldsymbol{\beta}, \mathbf{t})}{\partial \beta_{i}^{2}} .
$$

Thus, it is natural to define singular sectors in $\mathcal{R}_{q}$ as follows

Definition 4 Given a set of $2 q$ non negative integers $\mathbf{n}=\left(n_{1}, n_{2}, \cdots, n_{2 q}\right)$ with some $n_{i} \geq 1$, the singular sector $\operatorname{sing} \mathcal{R}_{q}(\mathbf{n})$ is defined as the set of points $\left(\boldsymbol{\beta}, \mathbf{t}^{(q)}\right) \in \mathcal{R}_{q}$ such that

$$
\left\{\begin{array}{l}
\frac{\partial^{k} \mathbb{W}}{\partial \beta_{i}^{k}}=0, \quad \forall 1 \leq k \leq n_{i}+1, \\
\frac{\partial^{n_{i}+2} \mathbb{W}}{\partial \beta_{i}^{n_{i}+2}} \neq 0,
\end{array}\right.
$$

for $i=1,2, \ldots, 2 q$. 
Spectral curves in gauge/string dualities: integrability, singular sectors and regularization. 24

Theorem 3 If a spectral curve in $\operatorname{sing} \mathcal{M}_{q}$ has roots $\beta_{i}$ with multiplicities $2 n_{i}+1$, then $\left(\boldsymbol{\beta}\left(\mathbf{t}^{(q)}\right), \mathbf{t}^{(q)}\right) \in \operatorname{sing} \mathcal{R}_{q}(\mathbf{n})$.

Proof From 90 and $\left(93\right.$ it is clear that at $\boldsymbol{\beta}=\boldsymbol{\beta}\left(\mathbf{t}^{(q)}\right)$ each derivative $\partial_{\beta_{i}}^{k+1} \mathbb{W}$ is proportional to the integral

$$
\begin{aligned}
& \oint_{\Gamma_{\infty}} \frac{y_{0}(z, \boldsymbol{\beta}) y(z, \mathbf{t})}{\left(z-\beta_{i}\right)^{k+1}} \frac{\mathrm{d} z}{2 \pi \mathrm{i}}=\oint_{\Gamma_{\infty}} \frac{\prod_{l=1}^{p}\left(z-\alpha_{l}\right) \prod_{j \neq i}\left(z-\beta_{j}\right)}{\left(z-\beta_{i}\right)^{k}} \frac{\mathrm{d} z}{2 \pi \mathrm{i}} \\
& =\left.\frac{1}{(k-1) !} \partial_{z}^{k-1}\left(\prod_{l=1}^{p}\left(z-\alpha_{l}\right) \prod_{j \neq i}\left(z-\beta_{j}\right)\right)\right|_{z=\beta_{i}} .
\end{aligned}
$$

Then the statement follows since, according to our assumption, the function $\prod_{l=1}^{p}\left(z-\alpha_{l}\right)$ has zeros of order $n_{i}$ at $z=\beta_{i}$.

As a consequence of this theorem we conclude that the sets $\operatorname{sing} \mathcal{R}_{q}(\mathbf{n})$ represent singular spectral curves in which double zeros $\alpha_{l}$ of $y(z)^{2}$ coalesce with simple zeros $\beta_{i}$. Figure 4 exhibits a process of this type in the cubic model.

\section{Critical behavior and the Multiple scaling limit method}

The observation made in the previous section drastically simplify the analysis of the singular behavior of spectral curves near branch points of multiplicity higher than one, as well as its possible regularization.

Let us consider a spectral curve such that at a certain value $\mathbf{t}_{0}^{(q)}$ the roots $\beta_{i 0}$ have multiplicities $2 n_{i}+1$, then at $\left(\boldsymbol{\beta}\left(\mathbf{t}_{0}^{(q)}\right), \mathbf{t}_{0}^{(q)}\right)$ we have

$$
\frac{\partial^{k} \mathbb{W}}{\partial \beta_{i}^{k}}=0, \quad \forall 1 \leq k \leq n_{i}+1, \quad \frac{\partial^{n_{i}+2} \mathbb{W}}{\partial \beta_{i}^{n_{i}+2}} \neq 0
$$

for $i=1, \ldots, 2 q$, and in virtue of 89 we also have

$$
\frac{\partial^{l_{1}+\ldots+l_{2 q} \mathbb{W}}}{\partial \beta_{1}^{l_{1}} \ldots \partial \beta_{2 q}^{l_{2 q}}}=0, \quad 1 \leq l_{i} \leq n_{i}+1 .
$$

To analyze the behavior of $\boldsymbol{\beta}\left(\mathbf{t}_{0}^{(q)}\right)$ near $\mathbf{t}_{0}^{(q)}=\left(t_{01}, \ldots, t_{0 q}\right)$ we will use the standard technique of the multiple scaling limit (see e.g. [26]). So, we take

$$
t_{k}=t_{0 k}+\varepsilon^{\alpha} t_{k}^{*}, \quad \beta_{i}=\beta_{i 0}+\varepsilon^{\gamma_{i}} \beta_{i}^{*},
$$

where $\varepsilon \ll 1$ and $\alpha$ and $\gamma_{i}$ are positive parameters to be determined. Now we expand $\mathbb{W}\left(\boldsymbol{\beta}, \mathbf{t}^{(q)}\right)$ near the point $\left(\boldsymbol{\beta}_{0}, \mathbf{t}_{0}^{(q)}\right)$. Writing $\mathbb{W}\left(\boldsymbol{\beta}, \mathbf{t}^{(q)}\right)=\mathbb{W}^{(0)}(\boldsymbol{\beta})+\sum_{k=1}^{q} t_{k} \mathbb{V}_{k}(\boldsymbol{\beta})$, taking into account (100), (101) and keeping the leading terms in the expansion, one gets 
Spectral curves in gauge/string dualities: integrability, singular sectors and regularization. 25

$$
\begin{aligned}
& \mathbb{W}\left(\boldsymbol{\beta}, \mathbf{t}^{(q)}\right)=\mathbb{W}_{0}+\varepsilon^{\alpha} \sum_{k=1}^{q} t_{k}^{*} A_{k}+\varepsilon^{\alpha} \sum_{k=1}^{q} \sum_{l=1}^{2 q} \varepsilon^{\gamma_{l}} t_{k}^{*} A_{k l} \beta_{l}^{*} \\
& +\varepsilon^{\alpha} \sum_{k=1}^{q} \sum_{l, m=1}^{2 q} \varepsilon^{\gamma_{l}+\gamma_{m}} t_{k}^{*} A_{k l m} \beta_{l}^{*} \beta_{m}^{*}+\sum_{k=1}^{2 q} \varepsilon^{\left(n_{k}+2\right) \gamma_{k}} B_{k}\left(\beta_{k}^{*}\right)^{n_{k}+2}+\ldots
\end{aligned}
$$

where

$$
\begin{aligned}
& \mathbb{W}_{0}=\mathbb{W}\left(\boldsymbol{\beta}_{0}, \mathbf{t}_{0}^{(q)}\right), \quad A_{k}=\mathbb{V}_{k}\left(\boldsymbol{\beta}_{0}\right), \quad A_{k l}=\frac{\partial \mathbb{V}_{k}}{\partial \beta_{l}}\left(\boldsymbol{\beta}_{0}\right), \\
& A_{k l m}=\frac{1}{2} \frac{\partial \mathbb{V}_{k}}{\partial \beta_{l} \partial \beta_{m}}\left(\boldsymbol{\beta}_{0}\right), \quad B_{k}=\frac{1}{\left(n_{k}+2\right) !} \frac{\partial^{n_{k}+2} \mathbb{W}}{\partial \beta_{k}^{n_{k}+2}}\left(\boldsymbol{\beta}_{0}, \mathbf{t}_{0}^{(q)}\right) .
\end{aligned}
$$

Consequently,

$$
\begin{aligned}
& \frac{\partial \mathbb{W}}{\partial \beta_{l}^{*}}=\varepsilon^{\alpha+\gamma_{l}} \sum_{k=1}^{q} t_{k}^{*} A_{k l}+\varepsilon^{\alpha+\gamma_{l}} 2 \sum_{k=1}^{q} \sum_{m=1}^{2 q} \varepsilon^{\gamma_{m}} t_{k}^{*} A_{k l m} \beta_{m}^{*} \\
& +\varepsilon^{\left(n_{l}+2\right) \gamma_{l}}\left(n_{l}+2\right) B_{l}\left(\beta_{l}^{*}\right)^{n_{l}+1}+\ldots .
\end{aligned}
$$

In the generic case when at least one $A_{k l}$ does not vanish, the second term is subdominant with respect to the first one. In such a case the balance between the first and third terms is achieved if $\alpha+\gamma_{l}=\left(n_{l}+2\right) \gamma_{l}$, i.e.

$$
\gamma_{l}=\frac{\alpha}{n_{l}+1}, \quad l=1, \ldots, 2 q .
$$

Thus, in the generic case the expansion of $\mathbb{W}\left(\boldsymbol{\beta}, \mathbf{t}^{(q)}\right)$ is of the form

$$
\mathbb{W}\left(\boldsymbol{\beta}, \mathbf{t}^{(q)}\right)=\mathbb{W}_{0}+\varepsilon \sum_{k=1}^{q} t_{k}^{*} A_{k}+\sum_{l=1}^{2 q} \varepsilon^{\frac{n_{l}+2}{n_{l}+1}}\left(\xi_{l} \beta_{l}^{*}+B_{l}\left(\beta_{l}^{*}\right)^{n_{l}+2}\right)+\ldots
$$

where $\xi_{l}=\sum_{k=1}^{q} t_{k}^{*} A_{k l}$ and we put $\alpha=1$.

Equations (103)-(104) imply that when $\mathbf{t}^{(q)}$ and $\boldsymbol{\beta}$ are approaching $\mathbf{t}_{0}^{(q)}$ and $\boldsymbol{\beta}_{0}$ then

$$
\beta_{l}-\beta_{l 0} \sim\left(\sum_{k=1}^{q}\left(t_{k}-t_{0 k}\right) A_{k l}\right)^{\frac{1}{n_{l}+1}}, \quad l=1, \ldots, 2 q .
$$

and hence the derivatives of $\beta_{l}$ blow up as

$$
\frac{\partial \beta_{l}}{\partial t_{k}} \sim\left(\sum_{k=1}^{q}\left(t_{k}-t_{0 k}\right) A_{k l}\right)^{-\frac{n_{l}}{n_{l}+1}}
$$

as $\mathbf{t}^{(q)} \rightarrow \mathbf{t}_{0}^{(q)}$.

An unbounded increase of derivatives of $\beta_{l}$ while approaching the singular sectors is the origin of the break of analyticity for the spectral curve and the free energy (8). 
Spectral curves in gauge/string dualities: integrability, singular sectors and regularization. 26

From the point of view of the hydrodynamic type equations associated to the differential equations (63), in particular for the Burgers-Hopf equations

$$
\frac{\partial \beta_{l}}{\partial t_{j}}=\beta_{l}^{N-j} \frac{\partial \beta_{l}}{\partial t_{N}}, \quad l=1, . ., N, \quad j=1, . ., N-1,
$$

associated with the equations (64), an unbounded increase of derivatives of $\beta_{l}$ represents the classical gradient catastrophe (see e.g. $[31,32]$ ). Within the theory of polynomials with multiple roots and singularities of $A_{N+1}$ type, this singular behavior is the analytical manifestation of the catastrophe arising in the transitions between two different strata (see e.g. [33 35]).

\section{Regularization of gradient catastrophes}

In all physical models where such catastrophe with derivatives happens it is regularized at the end by one or another mechanism. It is, for instance, dissipation or dispersion for hydrodynamical models (see e.g. ( [31])), quantum corrections or higher genus expansion in matrix models (see e.g. ([26])). Formally such mechanisms consist in the appearance of higher order derivatives in the basic relations or differential equations of the type (63) or (108). Concrete forms of these higher order corrections typically are obtained by appropriate expansion or limit for the known model exact relations or equations.

Here we suggest an approach for regularization which goes in the direction opposite to the standard ones. The idea is to deform the scheme presented above in such model independent manner that it naturally leads to the appearance of derivatives in equations (103) and (106) to prevents the blow up of $\partial_{t_{k}} \beta_{i}$. The principal point is the substitution of the equations (92) or (105) for critical points of the functions $\mathbb{W}$ by Euler-Lagrange equations

$$
\frac{\delta \mathbb{W}^{r e g}}{\delta \beta_{i}}=0, \quad i=1, \ldots, 2 q,
$$

where $\mathbb{W}^{\text {reg }}$ is an appropriate modification of $\mathbb{W}$ obtained by adding terms with derivatives. Thus we rewrite (105), dropping the insignificant second term, as

$$
\mathbb{W}\left(\boldsymbol{\beta}, \mathbf{t}^{(q)}\right)=\mathbb{W}_{0}+\varepsilon \sum_{l=1}^{2 q} \varepsilon^{\frac{1}{n_{l}+1}} \mathbb{U}_{l}\left(\beta_{l}^{*}\right)+\cdots
$$

where $\mathbb{U}_{l}\left(\beta_{l}^{*}\right)=\xi_{l} \beta_{l}^{*}+B_{l}\left(\beta_{l}^{*}\right)^{n_{l}+2}$. Modification of the polynomial 1110$)$ is achieved by adding terms with derivatives of the functions $\beta_{i}$ of appropriate order in $\varepsilon$. The general case is pretty involved and will be studied in a separate paper. Here we will consider some specific regularizations for particular situations when the corresponding equations (109) are rather simple.

In the case when all $n_{l}$ are different the terms in 110$)$ are of different degrees, so it is natural to treat each term in the sum (110) separately. The simplest nontrivial modification of $\mathbb{W}\left(\boldsymbol{\beta}, \mathbf{t}^{(q)}\right)$ is of the form

$$
\mathbb{W}^{r e g}\left(\boldsymbol{\beta}^{*}, \boldsymbol{\xi}\right)=\mathbb{W}_{0}+\varepsilon \sum_{l=1}^{2 q}\left(\varepsilon^{\frac{1}{n_{l}+1}} \mathbb{U}_{l}\left(\beta_{l}^{*}\right)+\varepsilon^{\delta_{l}} C_{l}\left(\frac{\partial \beta_{l}^{*}}{\partial \xi_{l}}\right)^{2}\right)+\cdots
$$


Spectral curves in gauge/string dualities: integrability, singular sectors and regularization. 27

with some constants $C_{l}$ and appropriate $\delta_{l}$. A balance of degrees in the Euler-Lagrange equation 109 or, equivalently, in the equation

$$
\frac{\delta \mathbb{W}^{r e g}}{\delta \beta_{i}^{*}}=\frac{\partial \mathbb{W}^{r e g}}{\partial \beta_{i}^{*}}-\sum_{k} \frac{\partial}{\partial \xi_{k}}\left(\frac{\partial \mathbb{W}^{r e g}}{\partial\left(\partial_{\xi_{k}} \beta_{i}^{*}\right)}\right)=0,
$$

implies that

$$
\delta_{l}=\frac{1}{n_{l}+1}
$$

Thus,

$$
\mathbb{W}^{r e g}\left(\boldsymbol{\beta}^{*}, \boldsymbol{\xi}\right)=\mathbb{W}_{0}+\sum_{l=1}^{2 q} \varepsilon^{\frac{n_{l}+2}{n_{l}+1}}\left(\xi_{l} \beta_{l}^{*}+B_{l}\left(\beta_{l}^{*}\right)^{n_{l}+2}+C_{l}\left(\partial_{\xi_{l}} \beta_{l}^{*}\right)^{2}\right)+\cdots
$$

and the Euler-Lagrange equations take the form

$$
2 C_{l} \frac{\partial^{2} \beta_{l}^{*}}{\partial \xi_{l}^{2}}=\xi_{l}+\left(n_{l}+2\right) B_{l}\left(\beta_{l}^{*}\right)^{n_{l}+1}, \quad l=1, \ldots, 2 q .
$$

In the case when $n_{l}=1$ for one of the equations $(112)$ then the simple redefinitions

$$
\beta_{l}^{*}=\left(\frac{16 C_{l}}{B_{l}^{3}}\right)^{\frac{1}{5}} \Omega, \quad \xi_{l}=\left(\frac{8 C_{l}^{2}}{B_{l}}\right)^{\frac{1}{5}} x
$$

convert this $l$-th equation into the Painlevè-I equation

$$
\frac{\partial^{2} \Omega}{\partial x^{2}}=6 \Omega^{2}+x
$$

This equation has appeared as the regularizing equation of different types of catastrophes (see e.g. [26, 36]). In our case the Tritronquèe solution of the Painlevè-I equation (113) describes the regularization of the blow up (107) with $n_{l}=1$, and the analyticity breaking associated with the formation of a third-order root from the merging of a double root $\alpha$ and the simple root $\beta_{l}$. A similar situation takes place for $n_{l} \geq 2$.

In the case when some of $n_{i}$ coincide, say, $n_{1}=n_{2}=\ldots=n_{k}=n$ the polynomial $\mathbb{W}$ is

$$
\begin{aligned}
& \mathbb{W}\left(\boldsymbol{\beta}, \mathbf{t}^{(q)}\right)=\mathbb{W}_{0}+\varepsilon^{\frac{n+2}{n+1}} \sum_{l=1}^{k}\left(\xi_{l} \beta_{l}^{*}+B_{l}\left(\beta_{l}^{*}\right)^{n+2}\right) \\
& +\varepsilon \sum_{l=k+1}^{2 q} \varepsilon^{\frac{1}{n_{l}+1}}\left(\xi_{l} \beta_{l}^{*}+B_{l}\left(\beta_{l}^{*}\right)^{n_{l}+2}\right)+\cdots
\end{aligned}
$$

The dominant contribution of $\beta_{1}^{*}, \beta_{2}^{*}, \ldots, \beta_{k}^{*}$ into $\mathbb{W}$ is of the same order. So, $\mathbb{W}^{r e g}\left(\boldsymbol{\beta}^{*}, \boldsymbol{\xi}\right)$ should naturally contains a mixture of derivatives of $\beta_{1}^{*}, \beta_{2}^{*}, \ldots, \beta_{k}^{*}$. Hence, the simplest natural ansatz for the modified $\mathbb{W}$ of 1114 would be 
Spectral curves in gauge/string dualities: integrability, singular sectors and regularization. 28

$$
\begin{aligned}
& \mathbb{W}^{r e g}\left(\boldsymbol{\beta}^{*}, \boldsymbol{\xi}\right)=\mathbb{W}_{0}+\varepsilon^{\frac{n+2}{n+1}}\left[\sum_{l=1}^{k}\left(\xi_{l} \beta_{l}^{*}+B_{l}\left(\beta_{l}^{*}\right)^{n+2}\right)\right. \\
& \left.+\frac{1}{2} \sum_{m, p, q=1}^{k} D_{m p q} \beta_{m}^{*} \frac{\partial \beta_{p}^{*}}{\partial \xi_{q}}+\frac{1}{2} \sum_{m, p, q, t=1}^{k} D_{m p q t} \frac{\partial \beta_{m}^{*}}{\partial \xi_{p}} \frac{\partial \beta_{q}^{*}}{\partial \xi_{t}}\right] \\
& +\varepsilon \sum_{l=k+1}^{2 q} \varepsilon^{\frac{1}{n_{l}+1}}\left(\xi_{l} \beta_{l}^{*}+B_{l}\left(\beta_{l}^{*}\right)^{n_{l}+2}+C_{l}\left(\frac{\partial \beta_{l}^{*}}{\partial \xi_{l}}\right)^{2}\right)+\cdots
\end{aligned}
$$

where $C_{l}, D_{m p q}, D_{m p q t}$ are some constants such that $D_{m p q}=-D_{p m q}, D_{m p q t}=D_{q t m p}$. The Euler-Lagrange equations corresponding to the variables $\beta_{1}^{*}, \ldots, \beta_{k}^{*}$ are

$$
\sum_{m, p, q=1}^{k} D_{i m p q} \frac{\partial^{2} \beta_{p}^{*}}{\partial \xi_{m} \partial \xi_{q}}-\sum_{m, p=1}^{k} D_{i m p} \frac{\partial \beta_{m}^{*}}{\partial \xi_{p}}=\xi_{i}+(n+2) B_{i}\left(\beta_{i}^{*}\right)^{n+1},
$$

while those corresponding to the variables $\beta_{k+1}^{*}, \ldots, \beta_{s}$ are the equations (112).

In the simplest case $k=2$ and under the constraints $D_{i m p q}=0$, the system 116 is equivalent to

$$
\begin{aligned}
& \frac{\partial \beta_{1}^{*}}{\partial \xi}=D_{122} \xi+C \eta+(n+2) B_{2} \beta_{2}^{* n+1}, \\
& \frac{\partial \beta_{2}^{*}}{\partial \xi}=-D_{121} \xi-A \eta-(n+2) B_{1} \beta_{1}^{* n+1},
\end{aligned}
$$

where the variables $\xi$ and $\eta$ are defined as $\xi_{1}=D_{121} \xi+A \eta, \xi_{2}=D_{122} \xi+C \eta$, with $A$ and $C$ being arbitrary constants. For $n=1$ and $A=C=0$ it is the special Riccati system. For $n=2$ and $A=C=0$ it is a particular instance of the differential cubic systems which can be viewed as the multi-component generalizations of the Abel equation (see e.g. 37|).

At $k=2$ and under the constraints $D_{m p q}=0$, with the change of variables $\xi_{1}=\xi+\eta$ $\xi_{2}=\xi-\eta$ and restricted to the subspace $\eta=0$ the system (116) takes the form

$$
\begin{aligned}
& \frac{\partial^{2} \beta_{1}^{*}}{\partial \xi^{2}}=A_{11} \beta_{1}^{* n+1}+A_{12} \beta_{2}^{* n+1}+A_{1} \xi, \\
& \frac{\partial^{2} \beta_{2}^{*}}{\partial \xi^{2}}=A_{21} \beta_{1}^{* n+1}+A_{22} \beta_{2}^{* n+1}+A_{2} \xi,
\end{aligned}
$$


Spectral curves in gauge/string dualities: integrability, singular sectors and regularization. 29

where

$$
\begin{aligned}
& A_{11}=\frac{4(n+2) B_{1} B_{22}}{B_{11} B_{22}-B_{12}^{2}}, \quad A_{12}=-\frac{4(n+2) B_{2} B_{12}}{B_{11} B_{22}-B_{12}^{2}}, \quad A_{1}=\frac{4\left(B_{22}-B_{12}\right)}{B_{11} B_{22}-B_{12}^{2}}, \\
& A_{21}=-\frac{4(n+2) B_{1} B_{12}}{B_{11} B_{22}-B_{12}^{2}}, \quad A_{22}=\frac{4(n+2) B_{2} B_{11}}{B_{11} B_{22}-B_{12}^{2}}, \quad A_{2}=\frac{4\left(B_{11}-B_{12}\right)}{B_{11} B_{22}-B_{12}^{2}}, \\
& B_{l m}=\sum_{i, j=1,2} D_{l i m j} .
\end{aligned}
$$

The system (116) for arbitrary $k$ also admits special reductions under which it takes a form similar to (118). The systems (116)-(118) describe regularizations of particular catastrophes associated with the singular sectors $\operatorname{sing} \mathcal{R}(\mathbf{n})$ such that $n_{1}=\ldots=n_{k}=n$.

Detailed study of the properties of such systems and their implications for the string and matrix theory will be presented in a separate publication.

\section{Acknowledgments}

L. Martínez Alonso and E. Medina are grateful to G. Álvarez for many useful conversations on the subject of spectral curves in gauge/string dualities. The financial support of the Universidad Complutense under project GR58/08-910556, the Comisión Interministerial de Ciencia y Tecnología under project FIS2011-22566 and PRIN 2008 grant no. 28002K9KXZ are gratefully acknowledged.

\section{Appendix A}

In this appendix we briefly discuss the elements of the theory of Abelian differentials in Riemann surfaces that we use in section 2.

Let $M$ be the genus $q-1$ Riemann surface associated to $(33)$, it is determined by two sheets

$$
M=M_{1} \cup M_{2}, \quad M_{i}=\left\{Q=\left(y_{0, i}(z), z\right)\right\},
$$

where

$$
y_{0,2}(z)=-y_{0,1}(z), \quad y_{0,1}(z) \sim z^{q}, \quad z \rightarrow \infty .
$$

We introduce the following Abelian differentials in $M$ :

(1) The canonical basis of first kind (i.e., holomorphic) Abelian differentials $\left\{\mathrm{d} \varphi_{i}\right\}_{i=1}^{q-1}$ with the normalization $A_{i}\left(\mathrm{~d} \varphi_{j}\right)=\delta_{i j}$. These differentials can be written as

$$
\mathrm{d} \varphi_{j}(z)=\frac{p_{j}(z)}{y_{0}(z)} \mathrm{d} z
$$

where the $p_{j}(z)$ are polynomials of degree not greater than $q-2$ uniquely determined by the normalization conditions. 
Spectral curves in gauge/string dualities: integrability, singular sectors and regularization. 30

(2) The second kind Abelian differentials $\mathrm{d} \Omega_{n}(n \geq 1)$ whose only poles are at $\infty_{1}$, such that

$$
\mathrm{d} \Omega_{n}(Q)=\left(n z^{n-1}+\mathcal{O}\left(z^{-2}\right)\right) \mathrm{d} z, \quad Q \rightarrow \infty_{1}, \quad z=z(Q),
$$

and normalization $A_{i}\left(\mathrm{~d} \Omega_{n}\right)=0(i=1, \ldots, q-1)$. It is easy to see that

$$
\mathrm{d} \Omega_{n}=\left(\frac{n}{2} z^{n-1}+\frac{P_{n}(z)}{y_{0}(z)}\right) \mathrm{d} z
$$

where the $P_{n}(z)$ are polynomials of the form

$$
P_{n}(z)=\frac{n}{2}\left(z^{n-1} y_{0}(z)\right)_{\oplus}+\sum_{i=0}^{q-2} c_{n i} z^{i}
$$

and the coefficients $c_{n i}$ are uniquely determined by the normalization conditions.

(3) The third kind Abelian differential $d \Omega_{0}$ whose only poles are at $\infty_{1}$ and $\infty_{2}$, such that

$$
\mathrm{d} \Omega_{0}(Q)= \begin{cases}\left(\frac{1}{z}+\mathcal{O}\left(z^{-2}\right)\right) \mathrm{d} z, & Q \rightarrow \infty_{1} \\ \left(-\frac{1}{z}+\mathcal{O}\left(z^{-2}\right)\right) \mathrm{d} z, & Q \rightarrow \infty_{2},\end{cases}
$$

and normalization $A_{i}\left(\mathrm{~d} \Omega_{0}\right)=0$ for all $i=1, \ldots, q-1$. It follows that

$$
\mathrm{d} \Omega_{0}=\frac{P_{0}(z)}{y_{0}(z)} \mathrm{d} z
$$

where $P_{0}(z)$ is a polynomial of the form

$$
P_{0}(z)=\left(z^{-1} y_{0}(z)\right)_{\oplus}+\sum_{i=0}^{q-2} c_{0 i} z^{i}
$$

and the coefficients $c_{0 i}$ are uniquely determined by the normalization conditions.

We notice that all the polynomials $p_{j}(z)$ and $P_{n}(z)$ also depend on the set of $2 q$ branch points $\boldsymbol{\beta}$, but for simplicity this dependence will not be indicated unless necessary.

\section{References}

[1] Cachazo F, Intriligator K and Vafa C 2001 Nuc. Phys. B 6033

[2] Dijkgraaf R and Vafa C 2002 Nuc. Phys. B 6443

[3] Dijkgraaf R and Vafa C 2002 Nuc. Phys. B 64421

[4] Seiberg N and Witten E 1994 Nuc. Phys. B 42619

[5] Cachazo F and Vafa C 2002 arXiv:hep-th/020601

[6] Deift P 1999 Orthogonal Polynomials and Random Matrices: A Riemann-Hilbert approach (Providence: American Mathematical Society)

[7] Bleher P 2008 Lectures on random matrix models. The Riemann-Hilbert approach (Amsterdam: North Holland)

[8] Álvarez G, Martínez Alonso L and Medina E 2010 J. Stat. Mech. Theory Exp. 03023

[9] Lazaroiu C I 2003 J. High Energy Phys. 03044

[10] Felder G and Riser R 2004 Nuc. Phys. B 691251 
Spectral curves in gauge/string dualities: integrability, singular sectors and regularization. 31

[11] Bilal A and Metzger S 2005 J. High Energy Phys. 08097

[12] Gonchar A and Rakhmanov E A 1987 Math. USSR Sbornik 62

[13] Gonchar A A and Rakhmanov E A 1989 Math. USSR Sbornik 62305

[14] Bleher P and Its A 1999 Ann. Math. 150 185-266

[15] Bertola M, Eynard B and Harnad J 2006 Commun. Math. Phys. 263401

[16] Bertola M and Mo M Y 2009 Adv. Math. 220154

[17] Bertola M 2011 Analysis and Math. Phys. 1167

[18] Martínez-Finkelshtein A and Rakhmanov E A 2011 Commun. Math. Phys. 30253

[19] Rakhmanov E A 2012 Orthogonal polynonials and s-curves Recent advances in orthogonal polynomials, special functions and their applications vol 578 of Contemp. Math. (Amer. Math. Soc. Providence, RI.) pp 195-239

[20] Álvarez G, Martínez Alonso L and Medina E 2013 J. High Energy Phys. 03170

[21] Ferrari F 2003 Phys. Rev. D 67085013

[22] Krichever I 1994 Comm.Pure Appl. Math. 47437

[23] Chekhov L and Mironov A 2003 Phys. Lett. B 552293

[24] Chekhov L, Marshakov A, Mironov A and Vasiliev D 2003 Phy. Lett. B 562323

[25] Darboux G 1915 Lecons sur la thèorie gènèrale des surfaces II surfaces II, (Gauthier Villars)

[26] Di Francesco P, Ginsparg P and Zinn-Justin J 1995 Phys. Rep. 254 1-133

[27] Konopelchenko B, Martínez Alonso L and Medina E 2011 Phys. Lett. A 375 867-872

[28] Mariño M, Pasquetti S and Putrov P 2010 J. High Energy Phys. 10074

[29] Bertola M and Tovbis A 2011 arXiv:1108.0321

[30] Givental A B 1982 Functional Analysis and its Applications 16 10-14

[31] Whitham G B 1974 Linear and nonlinear waves (J.Wiley and Sons, New-york)

[32] Rozdestvenski B and Yanenko N 1983 Systems of quasilinear their applications to gas dynamics (Math.Monog., v. 55, AMS, Providence, RI.)

[33] Arnold V I 1976 Comm.Pure Appl. Math. 29 557-582

[34] Arnold V I, Varchenko A N and Guseyn-Zade S M 1985 Singularities of differentiable maps (Birkhauser, Boston, Ma.)

[35] Thom R 1989 Structural stability and morphogenesis (Addison-Wesley, P.C. ,CA)

[36] Dubrovin B 2008 On universality of critical behaviour in hamiltonian pdes Geometry, topology and mathematical physics (AMS Transl., 224, Ser. 2, 59-109, AMS, Providence, RI)

[37] Murphy G 1960 Ordinary differential equations and their solutions (Van Vostrand,) 